\title{
Aerodynamic Shape Optimization Investigations of the Common Research Model Wing Benchmark
}

\author{
Zhoujie Lyu, $\stackrel{*}{-}$ Gaetan K. W. Kenway,, \pm and Joaquim R. R. A. Martins \\ University of Michigan, Ann Arbor, Michigan 48109
}

\begin{abstract}
DOI: $10.2514 / 1 . J 053318$
Despite considerable research on aerodynamic shape optimization, there is no standard benchmark problem allowing researchers to compare results. This work addresses this issue by solving a series of aerodynamic shape optimization problems based on the Common Research Model wing benchmark case defined by the Aerodynamic Design Optimization Discussion Group. The aerodynamic model solves the Reynolds-averaged Navier-Stokes equations with a Spalart-Allmaras turbulence model. A gradient-based optimization algorithm is used in conjunction with an adjoint method that computes the required derivatives. The drag coefficient is minimized subject to lift, pitching moment, and geometric constraints. A multilevel technique is used to reduce the computational cost of the optimization. A single-point optimization is solved with 720 shape variables using a 28.8 -million-cell mesh, reducing the drag by $8.5 \%$. A more realistic design is achieved through a multipoint optimization. Multiple local minima are found when starting from multiple randomly generated geometries, but the minimum drag values are within $0.1 \mathrm{drag}$ counts of each other, and the geometries differ by only $0.4 \%$ of the mean aerodynamic chord. The effect of varying the number of shape design variables is examined. The Common Research Model wing benchmark problem proved to be useful for evaluating our design optimization framework, and the geometries and meshes for both the baseline and optimized wings are available as supplemental materials in this paper.
\end{abstract}

\section{Introduction}

$\mathbf{T}$ HE design of transonic transport aircraft wings is particularly important because of the large number of such aircraft operating on a daily basis and because small changes in the wing shape may have a large impact on fuel burn. This directly affects both the airlines' cash operating cost and the emission of greenhouse gases.

Advances in high-performance computing hardware and algorithms have enabled the ever-increasing fidelity of the computational fluid dynamics (CFD) models used for evaluating aircraft performance. As the computational time for a given CFD model reduces below a certain level, it becomes feasible to use it together with numerical optimization to perform aircraft design. Although there are various possible optimization techniques, the use of gradient-based algorithms together with an adjoint method that computes the required gradients efficiently has proven to be particularly effective. Such optimizations typically require a total time equivalent to $\mathcal{O}\left(10^{2}\right)$ CFD simulations to obtain optimal designs. This enables wing designers to shorten design cycle times and thus explore the design space more effectively. They can also obtain detailed designs earlier in the design process, allowing them to better understand the design tradeoffs and to make more informed design decisions.

Aerodynamic shape optimization can be dated back to the 16th century, when Newton [1] used calculus of variations to minimize the fluid drag of a body of revolution with respect to the body's shape. Although there were many significant developments in optimization theory after that, it was only in the 1960s that both the theory and the computer hardware became advanced enough to make numerical

Presented as Paper 2014-0567 at the 52nd AIAA Aerospace Sciences Meeting, National Harbor, MD, 13-17 January 2014; received 31 December 2013; revision received 19 May 2014; accepted for publication 26 May 2014; published online 15 October 2014. Copyright @ 2014 by Zhoujie Lyu, Gaetan Kenway, and Joaquim R. R. A. Martins. Published by the American Institute of Aeronautics and Astronautics, Inc., with permission. Copies of this paper may be made for personal or internal use, on condition that the copier pay the $\$ 10.00$ per-copy fee to the Copyright Clearance Center, Inc., 222 Rosewood Drive, Danvers, MA 01923; include the code 1533-385X/14 and \$10.00 in correspondence with the CCC.

*Ph.D. Candidate, Department of Aerospace Engineering. Student Member AIAA.

${ }^{\dagger}$ Postdoctoral Research Fellow, Department of Aerospace Engineering. Member AIAA.

${ }^{\ddagger}$ Associate Professor, Department of Aerospace Engineering. Associate Fellow AIAA. optimization a viable tool for everyday applications. The application of gradient-based optimization to aerodynamic shape optimization was pioneered in the 1970s. The aerodynamic analysis at the time was a full-potential small perturbation inviscid model, and the gradients were computed using finite differences. Hicks et al. [2] first tackled airfoil design optimization problems. Hicks and Henne [3] then used a three-dimensional solver to optimize a wing with respect to 11 design variables representing both airfoil shape and the twist distribution.

Because small local changes in wing shape have a large effect on performance, wing design optimization is especially effective for large numbers of shape variables. As the number of design variables increases, the cost of computing gradients with finite-differences becomes prohibitive. The development of the adjoint method addressed this issue, enabling the computation of gradients at a cost independent of the number of design variables. For a review of methods for computing aerodynamic shape derivatives, including the adjoint method, see Peter and Dwight [4]. For a generalization of the adjoint method and its connection to other methods for computing derivatives, see Martins and Hwang [5].

Pironneau [6] pioneered the adjoint approach by deriving the adjoint of the Stokes equations and the incompressible Euler equations [7] to optimize airfoil profiles. Jameson [8] extended the adjoint method to handle inviscid compressible flows, making it suitable for the design of transonic airfoils. Since then, adjoint implementations for the compressible Euler equations have been used by various researchers to perform aerodynamic shape optimization. Reuther et al. [9, 10], for example, performed the aerodynamic shape optimization of complete aircraft configurations. The development of more robust CFD mesh deformations has made it possible to widen the range of the design parameters and study, for example, nonplanar geometries [11,12].

The aerodynamic design of transonic wings requires a model that can represent the shock-wave boundary-layer interaction because there is a strong nonlinear coupling between airfoil shape, wave drag, and viscous effects. Therefore, using a model that relies solely on the Euler equations is insufficient and can even be misleading [13].

Fortunately, the adjoint method has been extended to the compressible Navier-Stokes equations with turbulence models, enabling us to tackle practical aerodynamic design problems. Jameson et al. [14] optimized a wing-body configuration modeled with the compressible Navier-Stokes equations using a continuous adjoint approach. They used a 590,000-cell mesh and achieved a shock-free solution at Mach 0.86. Anderson and Bonhaus [15] optimized airfoil 


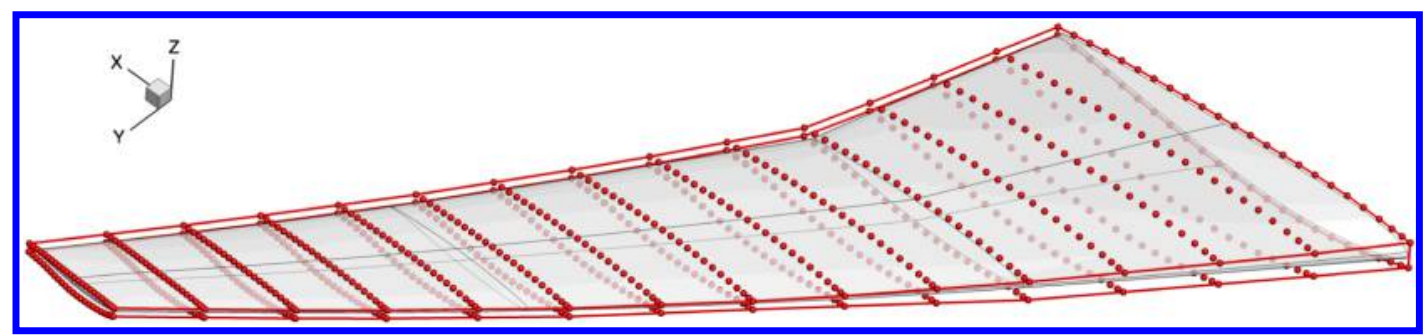

Fig. 1 Shape design variables are the $z$ displacements of 720 FFD control points shown as spheres.

shapes using a discrete adjoint that included the linearization of the Spalart-Allmaras turbulence model. Nielsen and Anderson [16] further extended the approach to the three-dimensional Reynoldsaveraged Navier-Stokes equations. They achieved an $8 \%$ drag reduction for the ONERA M6 wing with thickness and camber design variables at two chordwise locations. Dwight and Brezillon [17] and Brezillon and Dwight [18] optimized the DLR-F6 wing-body configuration using a Reynolds-averaged Navier-Stokes (RANS) solver and a discrete adjoint, achieving a 10-count drag reduction by varying 96 design variables.

Lyu et al. [13] developed a discrete adjoint for the RANS equations and Spalart-Allmaras turbulence model using algorithmic differentiation to construct the required derivative terms. They used this adjoint implementation to perform aerodynamic shape optimizations of the ONERA M6 wing with 192 design variables for both the Euler and RANS models. They observed significant differences between the optimal shapes obtained with Euler and RANS, which emphasized the importance of including the viscous compressible effects in transonic aerodynamic shape design.

The efforts mentioned previously use aerodynamic shape optimization frameworks combining different CFD solvers, adjoint implementations, optimizers, and geometry parameterizations, all applied to different design optimization problems. Thus, we need a set of benchmark cases for aerodynamic design optimization, following a model similar to that of the Drag Prediction Workshop [19-21]. To address this issue, a few researchers formed the Aerodynamic Design Optimization Discussion Group (ADODG) and developed four benchmark problems to test aerodynamic optimization methods. These problems range from the optimization of a two-dimensional airfoil using the Euler equations to threedimensional shape optimization using the RANS equations.

In this paper, we address the lack of benchmarks in aerodynamic design optimization by presenting a comprehensive set of results for what is currently the most computationally intensive benchmark problem among the test cases: the lift-constrained drag minimization of the NASA Common Research Model (CRM) wing with a RANS model. Four papers, including this one, were presented on this test case at the 2014 AIAA Science and Technology Forum and Exposition in a special session organized by the ADODG [22-25].

Our optimized geometries and meshes for this case are available as supplemental materials (Supplemental Data S1-S8) for this paper. We also solve two additional problems for the same wing that are not currently part of the benchmark: a multipoint case, and a case with more restrictive thickness constraints. In addition, we study the effect of the grid size, the number of shape design variables, and their distributions. We also demonstrate the robustness of our aerodynamic shape optimization framework by starting the optimization from a random perturbation of the CRM wing geometry.

We developed a multilevel optimization acceleration technique to increase the performance of the aerodynamic shape optimization. This method is analogous to the grid sequencing or the multigrid startup strategy often used in CFD. Using this method, aerodynamic shape optimization with a large mesh size requires significantly less computational time.

The majority of the aerodynamic shape optimization problems in the literature are solved with gradient-based optimizers

${ }^{\S}$ Data available online at https://info.aiaa.org/tac/ASG/APATC/ AeroDesignOpt-DG/default.aspx [retrieved May 2014].
$[9,10,12,26,27]$. High-fidelity aerodynamic shape optimization with a large number of design variables has the potential to have multiple local minima. The problem is that, because of the high number of dimensions and the high cost of the function evaluations, the design space is impossible to visualize fully. This makes it challenging to estimate the number of local minima and to form a complete picture of the design space. Several authors explored the multimodality in aerodynamic shape optimization with gradient-free optimization $[28,29]$ and combinations of gradient-free and gradient-based optimization [30]. However, there has been no thorough study for RANS-based three-dimensional aerodynamic shape optimization with large numbers of shape variables. In this paper, we explore multimodality by performing several shape optimizations starting from randomly generated geometries.

The paper is organized as follows. The numerical tools used in this work are described in Sec. II. The problem formulation, the mesh, and the baseline geometry are described in Sec. III. The aerodynamic shape optimization of the CRM wing is presented in Sec. IV, and the multilevel optimization acceleration technique is discussed in Sec. V. In Sec. VI, the robustness of the approach is demonstrated by the optimization of a wing with a randomly perturbed surface. The effect of the number of design variables is investigated in Sec. VII. A more restrictive thickness constraint is examined in Sec. VIII, and multipoint optimization is considered in Sec. IX.

\section{Methodology}

This section describes the numerical tools and methods that we used for the shape optimization studies. These tools are components of the framework for multidisciplinary design optimization (MDO) of aircraft configurations with high fidelity (MACH) [31]. MACH can perform the simultaneous optimization of aerodynamic shape and structural sizing variables considering aeroelastic deflections [32]. However, in this paper, we use only the components of $\mathrm{MACH}$ that are relevant for aerodynamic shape optimization: the geometric parameterization, mesh perturbation, CFD solver, and optimization algorithm.

\section{A. Geometric Parameterization}

We use a free-form deformation (FFD) volume approach to parameterize the wing geometry [33]. The FFD volume parameterizes the geometry changes rather than the geometry itself, resulting in a more efficient and compact set of geometry design variables, thus making it easier to manipulate complex geometries. Any geometry may be embedded inside the volume by performing a Newton search to map the parameter space to the physical space. All the geometric changes are performed on the outer boundary of the FFD volume. Any modification of this outer boundary indirectly modifies the embedded objects. The key assumption of the FFD approach is that the geometry has constant topology throughout the optimization process, which is usually the case in wing design. In addition, because FFD volumes are trivariate B-spline volumes, the derivatives of any point inside the volume can be easily computed. Figure 1 shows the FFD volume and the geometric control points used in the aerodynamic shape optimization. The shape design variables are the displacement of all FFD control points in the vertical $(z)$ direction. 


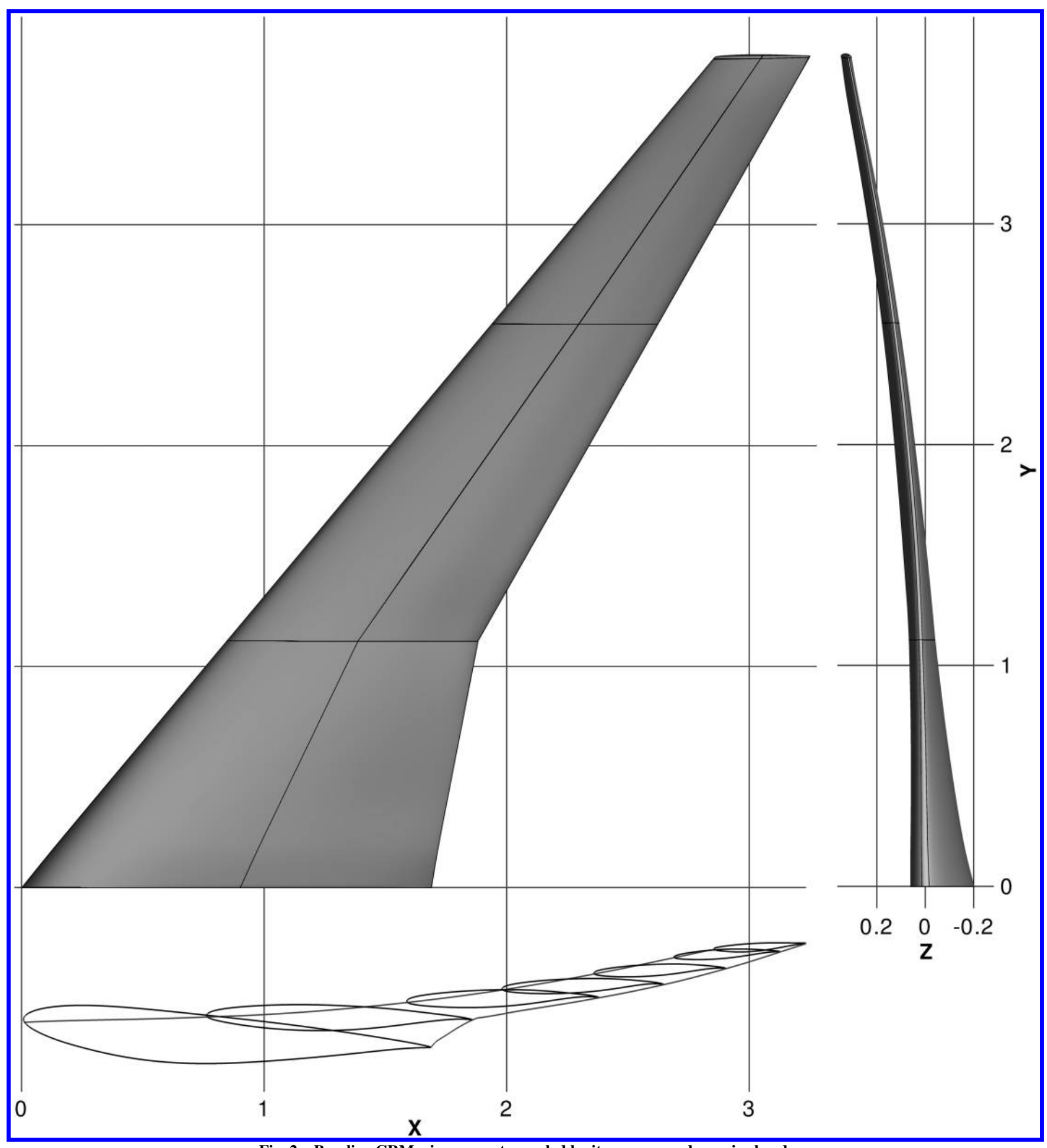

Fig. 2 Baseline CRM wing geometry scaled by its mean aerodynamic chord.

\section{B. Mesh Perturbation}

Because FFD volumes modify the geometry during the optimization, we must perturb the mesh for the CFD to solve for

Table 1 Mesh-convergence study for the baseline CRM wing

\begin{tabular}{lccccc}
\hline \hline Mesh level & Mesh size & $C_{D}$ & $C_{L}$ & $C_{M}$ & $\alpha, \mathrm{deg}$ \\
\hline$h=0$ & $\infty$ & 0.01990 & & & \\
L00 & $230,686,720$ & 0.01992 & 0.5000 & -0.1776 & 2.2199 \\
L0 & $28,835,840$ & 0.01997 & 0.5000 & -0.1790 & 2.2100 \\
L1 & $3,604,480$ & 0.02017 & 0.5000 & -0.1810 & 2.1837 \\
L2 & 450,560 & 0.02111 & 0.5000 & -0.1822 & 2.1944 \\
\hline \hline
\end{tabular}

the modified geometry. The mesh perturbation scheme used in this work is a hybridization of algebraic and linear elasticity methods, developed by Kenway et al. [33]. The idea behind the hybrid scheme is to apply a linear-elasticity-based perturbation scheme to a coarse approximation of the mesh to account for large, low-frequency perturbations, and to use the algebraic warping approach to attenuate small, high-frequency perturbations. For the results in this paper, the additional robustness of the hybrid scheme is not required, and so we use only the algebraic scheme.

\section{Computational-Fluid-Dynamics Solver}

We use SUmb [34] as the CFD solver; it is a finite-volume, cellcentered multiblock solver for the compressible Euler, laminar 


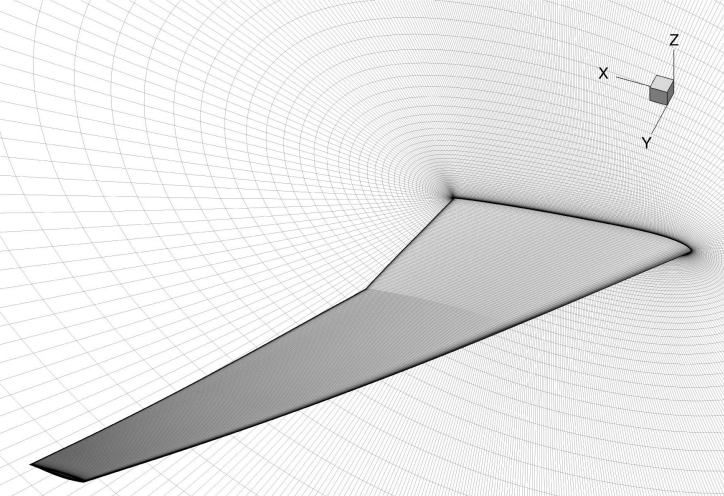

a) L0 mesh: 28.8 million cells, 199.7 drag counts

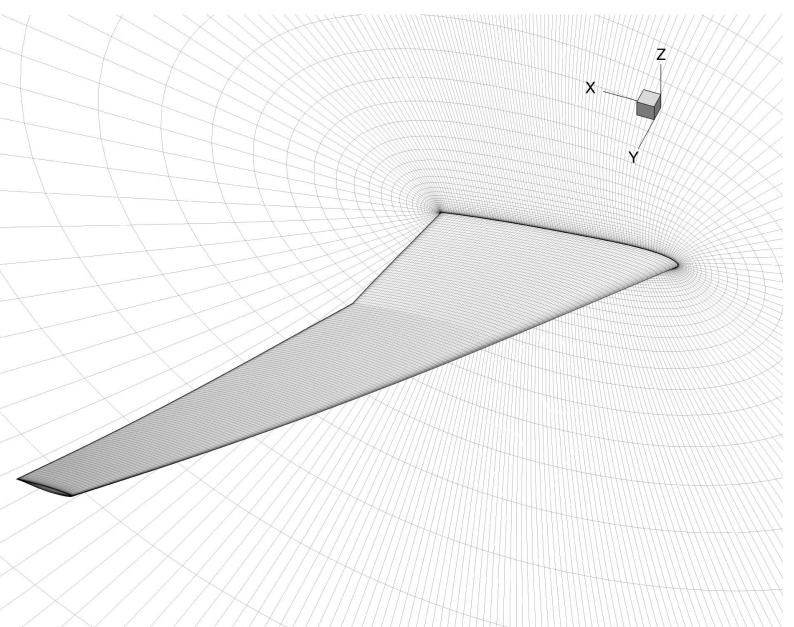

b) L1 mesh: 3.6 million cells, 201.7 drag counts

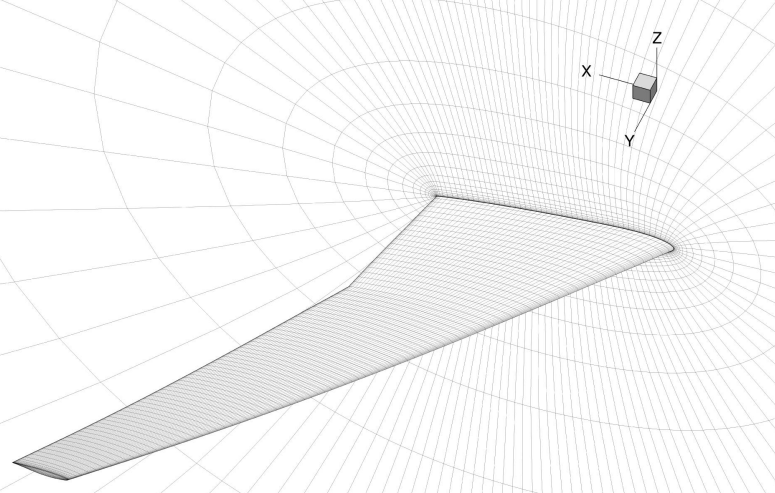

c) $L 2$ mesh: $450 \mathrm{k}$ cells, 211.1 drag counts

Fig. $3 O$-grids of varying sizes were generated using a hyperbolic mesh generator.

Navier-Stokes and RANS equations (steady, unsteady, and timeperiodic). SUmb provides options for a variety of turbulence models with one, two, or four equations and options for adaptive wall functions. The Jameson-Schmidt-Turkel scheme [35] augmented with artificial dissipation is used for the spatial discretization. The main flow is solved using an explicit multistage Runge-Kutta method, along with geometric multigrid. A segregated Spalart-
Allmaras turbulence equation is iterated with the diagonally dominant alternating-direction implicit method.

To efficiently compute the gradients required for the optimization, we have developed and implemented a discrete adjoint method for the Euler and RANS equations within SUmb [13,36]. The adjoint implementation supports both the full-turbulence and frozenturbulence modes, but in the present work, we use the full-turbulence adjoint exclusively. We solve the adjoint equations with preconditioned generalized minimal residual method (GMRES) [37] using PETSc [38-40]. We have previously performed extensive Euler-based aerodynamic shape optimization $[41,42]$ and aerostructural optimization $[32,43]$. However, we have observed serious issues with the resulting optimal Euler-based designs due to the missing viscous effects. Although Euler-based optimization can provide design insights, we found that the resulting optimal Euler shapes are significantly different from those obtained with RANS [13]. Euleroptimized shapes tend to exhibit a sharp pressure recovery near the trailing edge, which is nonphysical because such flow near the trailing edge would actually separate. Thus, RANS-based shape optimization is necessary to achieve realistic designs.

\section{Optimization Algorithm}

Because of the high computational cost of CFD solutions, we must choose an optimization algorithm that requires a reasonably low number of function evaluations. Gradient-free methods, such as genetic algorithms, have a higher probability of getting close to the global minimum for multimodal functions. However, slow convergence and the large number of function evaluations make gradient-free aerodynamic shape optimization infeasible with the current computational resources, especially for large numbers of design variables. Because we require hundreds of design variables, we use a gradientbased optimizer combined with adjoint gradient evaluations to solve the problem efficiently.

The optimization algorithm we use for all the results presented herein is SNOPT (from "sparse nonlinear optimizer") [44] through the Python interface pyOpt [45]. SNOPT is a gradient-based optimizer that implements a sequential quadratic programming method; it is capable of solving large-scale nonlinear optimization problems with thousands of constraints and design variables. SNOPT uses a smooth augmented Lagrangian merit function, and the Hessian of the Lagrangian is approximated using a limited-memory quasi-Newton method.

\section{Problem Formulation}

The goal of this optimization case is to perform lift-constrained drag minimization of the NASA CRM wing using the RANS equations. In this section, we provide a complete description of the problem.

\section{A. Baseline Geometry}

The baseline geometry is a wing with a blunt trailing edge extracted from the CRM wing-body geometry [20,21]. The NASA CRM geometry was developed for applied CFD validation studies. The CRM is representative of a contemporary transonic commercial transport, with a size similar to that of a Boeing 777. The CRM has 3.5 more quarter-chord wing sweep and $10.3 \%$ less wing area than the Boeing 777-200 [46]. The CRM geometry has been optimized in aerodynamic performance. However, several design features, such as an aggressive pressure recovery in the outboard wing, were introduced into the design to make it more interesting for research purposes and to protect intellectual property. This baseline geometry provides a reasonable starting point for the optimization, while leaving room for further performance improvements. In addition, the CRM was designed together with the fuselage of the full CRM configuration, and so its performance is degraded when only the wing is considered.

The geometry and specifications are given by the ADODG, and we repeat them here for convenience. The fuselage and tail are

"Data available online at https://info.aiaa.org/tac/ASG/APATC/ AeroDesignOpt-DG/default.aspx [retrieved May 2014]. 
Table 2 Aerodynamic shape optimization problem

\begin{tabular}{lllr}
\hline \hline & Function/variable & Description & Quantity \\
\hline Minimize & $C_{D}$ & Drag coefficient & \\
& & & \\
With respect to & $\alpha$ & Angle of attack & 1 \\
& $z$ & FFD control point $z$ coordinates & 720 \\
& & Total design variables & 721 \\
Subject to & $C_{L}=0.5$ & Lift coefficient constraint & 1 \\
& $C_{M_{y} \geq-0.17}$ & Moment coefficient constraint & 1 \\
& $t \geq 0.25 t_{\text {base }}$ & Minimum thickness constraints & 750 \\
& $V \geq V_{\text {base }}$ & Minimum volume constraint & 1 \\
& $\Delta z_{\mathrm{TE}, \text { upper }}=-\Delta z_{\mathrm{TE}, \text { lower }}$ & Fixed trailing-edge constraints & 15 \\
& $\Delta z_{\mathrm{LE}, \text { upper, root }}=\Delta z_{\mathrm{LE}, \text { lower, root }}$ & Fixed-wing root incidence constraint & 1 \\
& & Total constraints & 769 \\
\hline \hline
\end{tabular}

removed from the original CRM, and the root of the remaining wing is moved to the symmetry plane. This baseline geometry is shown in Fig. 2. All coordinates are scaled by the mean aerodynamic chord ( $275.8 \mathrm{in}$.). The resulting reference chord is 1.0 , and the half span is 3.758151. The moment reference point is at $(x, y, z)=$ $(1.2077,0.0,0.007669)$, whereas the reference area is 3.407014 . The baseline geometry is available in Supplemental Data S1.

\section{B. Mesh-Convergence Study}

We generate the mesh for the CRM wing using an in-house hyperbolic mesh generator. The mesh is marched out from the surface mesh using an $O$-grid topology to a far field located at a distance of 25 times the span (about 185 mean chords). The nominal cruise flow condition is Mach 0.85 with a Reynolds number of 5 million based on the mean aerodynamic chord. The mesh we generated for the test case optimization contains 28.8 million cells. The mesh size and $y_{\max }^{+}$ values under the nominal operating condition are listed in Table 1 .

We perform a mesh-convergence study to determine the resolution accuracy of this mesh. Table 1 lists the drag and moment coefficients for the baseline meshes. We also compute the zero-grid spacing drag using Richardson's extrapolation, which estimates the drag value as the grid spacing approaches zero [47]. The zero-grid spacing drag coefficient is 199.0 counts for the baseline CRM wing. We can see that the L0 mesh has sufficient accuracy; the difference in the drag coefficient for the L0 mesh and the zero-grid spacing drag is within one drag count. The surface and symmetry plane meshes for the $\mathrm{LO}$, L1, and L2 grid levels are shown in Fig. 3 . These meshes are available in Supplemental Data $\underline{\mathrm{S}}-\underline{\mathrm{S}} 4$ in the $\overline{\mathrm{CFD}}$ general notation system format.

\section{Optimization Problem Formulation}

The aerodynamic shape optimization seeks to minimize the drag coefficient by varying the shape design variables subject to a lift constraint $\left(C_{L}=0.5\right)$ and a pitching moment constraint $\left(C_{M_{y}} \geq\right.$ $-0.17)$. The shape design variables are the $z$-coordinate movements of 720 control points on the FFD volume (shown in Fig. 1) and the angle of attack. The control points at the trailing edge are constrained to avoid any movement of the trailing edge. Therefore, the twist about the trailing edge can be implicitly altered by the optimizer using the remaining degrees of freedom. The leadingedge control points at the wing root are also constrained to maintain a constant incidence for the root section. There are 750 thickness

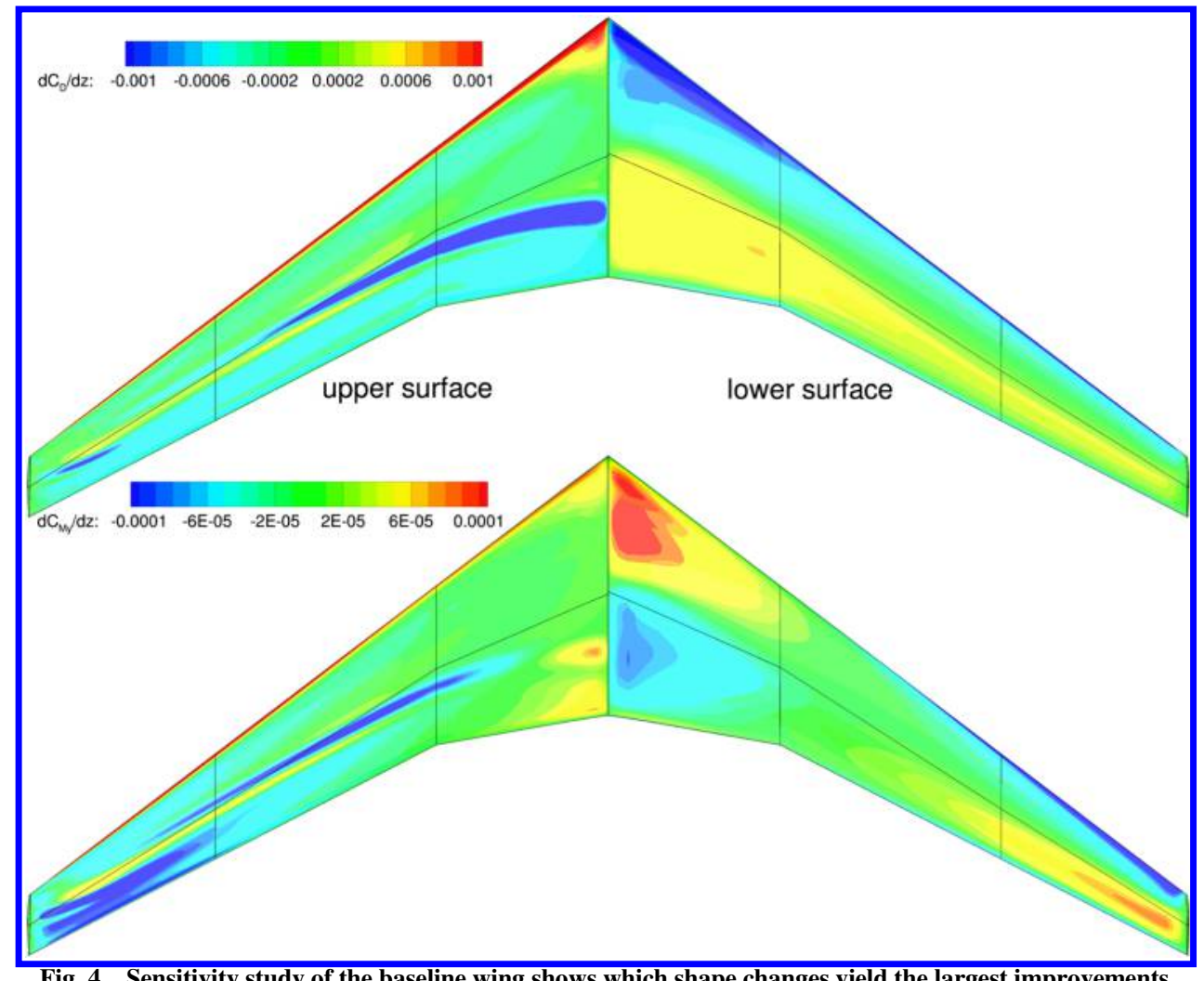

Fig. 4 Sensitivity study of the baseline wing shows which shape changes yield the largest improvements. 


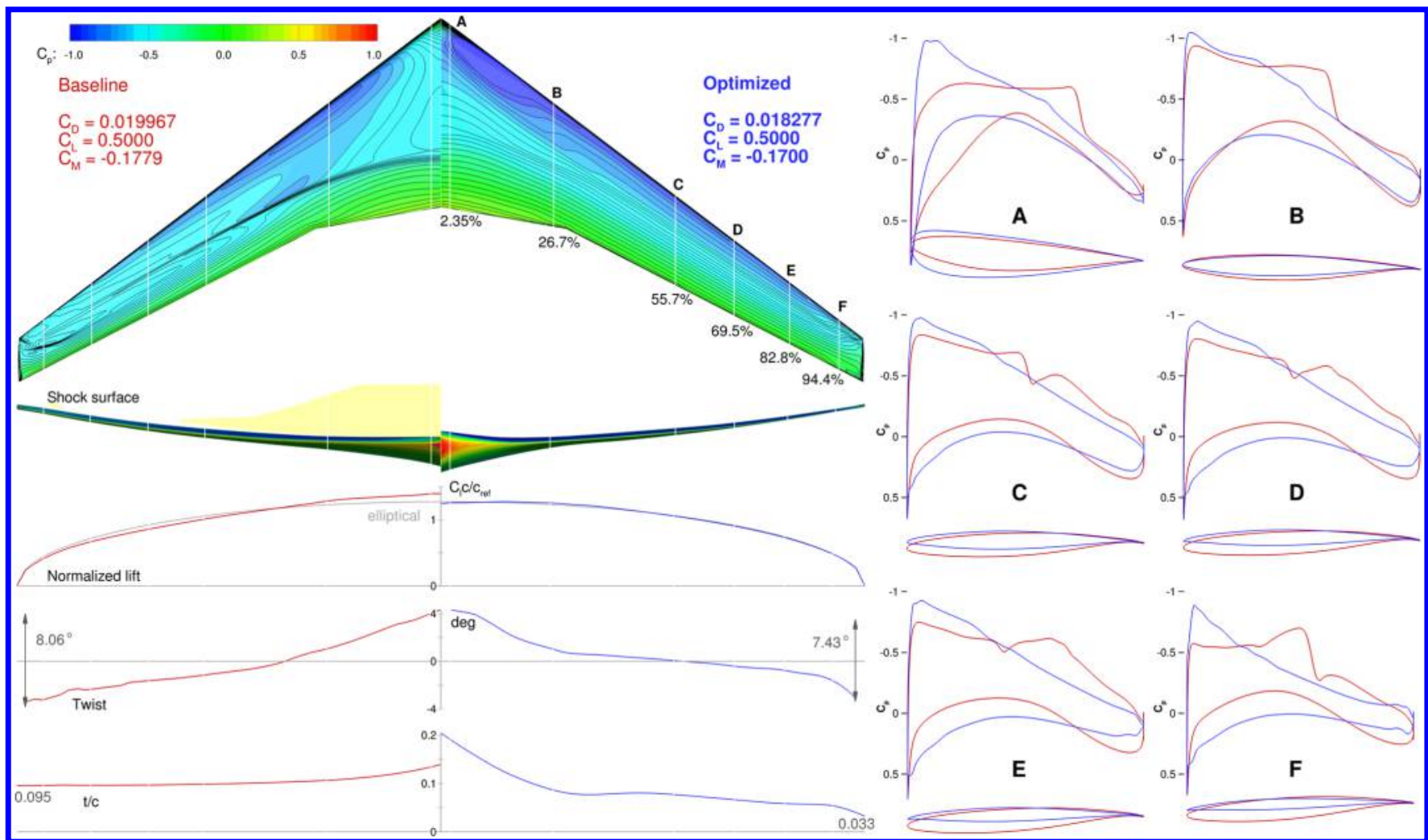

Fig. 5 The optimized wing is shock-free and has $8.5 \%$ lower drag.

constraints imposed in a 25 chordwise and 30 spanwise grid covering the full span and from 1 to $99 \%$ local chord. The thickness is set to be greater than $25 \%$ of the baseline thickness at each location. Finally, the internal volume is constrained to be greater than or equal to the baseline volume. The complete optimization problem is described in Table 2 .

\section{Surface Sensitivity on the Baseline Geometry}

To examine the potential improvements of the baseline geometry, we performed a sensitivity analysis. The sensitivity of the drag and pitching moment with respect to the airfoil shape is shown in Fig. 4 as a contour plot of the derivatives of $C_{D}$ and $C_{M_{y}}$ with respect to shape variations in the $z$ direction. The regions with the highest gradient of $C_{D}$ are near the shock on the upper surface and near the wing leading edge. This indicates that leading-edge shaping and shock reduction through local shape changes should be the major drivers in $C_{D}$ reduction at the beginning of the optimization. As for $C_{M_{y}}$, the shape changes near the root and tip of the wing are the most effective in adjusting the pitching moment. Because these sensitivity plots are a linearization about the current design point, they provide no information about the constraints. Nonetheless, these sensitivity plots indicate what drives the design at this design point.

\section{Single-Point Aerodynamic Shape Optimization}

In this section, we present our aerodynamic design optimization results for the CRM wing benchmark problem (described in Table 2) under the nominal flight condition (Mach $0.85, R e=5 \times 10^{6}$ ). We use the L0 grid (28.8 million cells) for the optimization, thanks to a multilevel optimization acceleration technique that significantly reduces the overall computational cost of the optimization. The details of this technique are presented in Sec. V. Our optimization procedure reduced the drag from 199.7 to 182.8 counts (i.e., an $8.5 \%$ reduction). The corresponding Richardson-extrapolated zero-grid spacing drag decreased from 199.0 to 181.9 counts. Given that the CRM configuration was designed by experienced aerodynamicists, this is a significant improvement (although they designed the wing in the presence of the fuselage, which we are ignoring in this problem). The optimized geometry and meshes are available in Supplemental Data S6-S8. A movie of the optimization iteration history for this case is available in Supplemental Data S9.

Figure 5 shows a detailed comparison of the baseline wing and the optimized wing. In this figure, the baseline wing results are shown on the left, and the optimized wing results are shown on the right. At the optimum, the lift coefficient target is met, and the pitching moment is reduced to the lowest allowed value. The lift distribution of the optimized wing is much closer to the elliptical distribution than that of the baseline, indicating an induced drag that is close to the theoretical minimum for a planar wake. This is achieved by finetuning the twist distribution and airfoil shapes. The baseline wing has a near-linear twist distribution. The optimized design has more twist at the root and tip and less twist near midwing. The overall twist angle changed only slightly, from 8.06 to $7.43 \mathrm{deg}$.

The optimized thickness distribution is significantly different from that of the baseline because the thicknesses are allowed to decrease to $25 \%$ of the original thickness, and there is a strong incentive to reduce the airfoil thicknesses to reduce wave drag. The volume is

Table 3 Drag differences between baseline and optimized meshes are consistent for each level

\begin{tabular}{lccccccc}
\hline \hline Mesh level & Mesh size & Baseline $C_{D}$ & Optimized $C_{D}$ & $\Delta C_{D}$ & $C_{L}$ & Optimized $C_{M}$ & Optimized $\alpha$, deg \\
\hline$h=0$ & $\infty$ & 0.01990 & 0.01819 & 0.00171 & & & \\
L00 & $230,686,720$ & 0.01992 & 0.01820 & 0.00171 & 0.5000 & -0.1694 & 2.1759 \\
L0 & $28,835,840$ & 0.01997 & 0.01825 & 0.00172 & 0.5000 & -0.1700 & 2.1660 \\
L1 & $3,604,480$ & 0.02017 & 0.01846 & 0.00171 & 0.5000 & -0.1710 & 2.1584 \\
L2 & 450,560 & 0.02111 & 0.01964 & 0.00147 & 0.5000 & -0.1731 & 2.1970 \\
\hline \hline
\end{tabular}



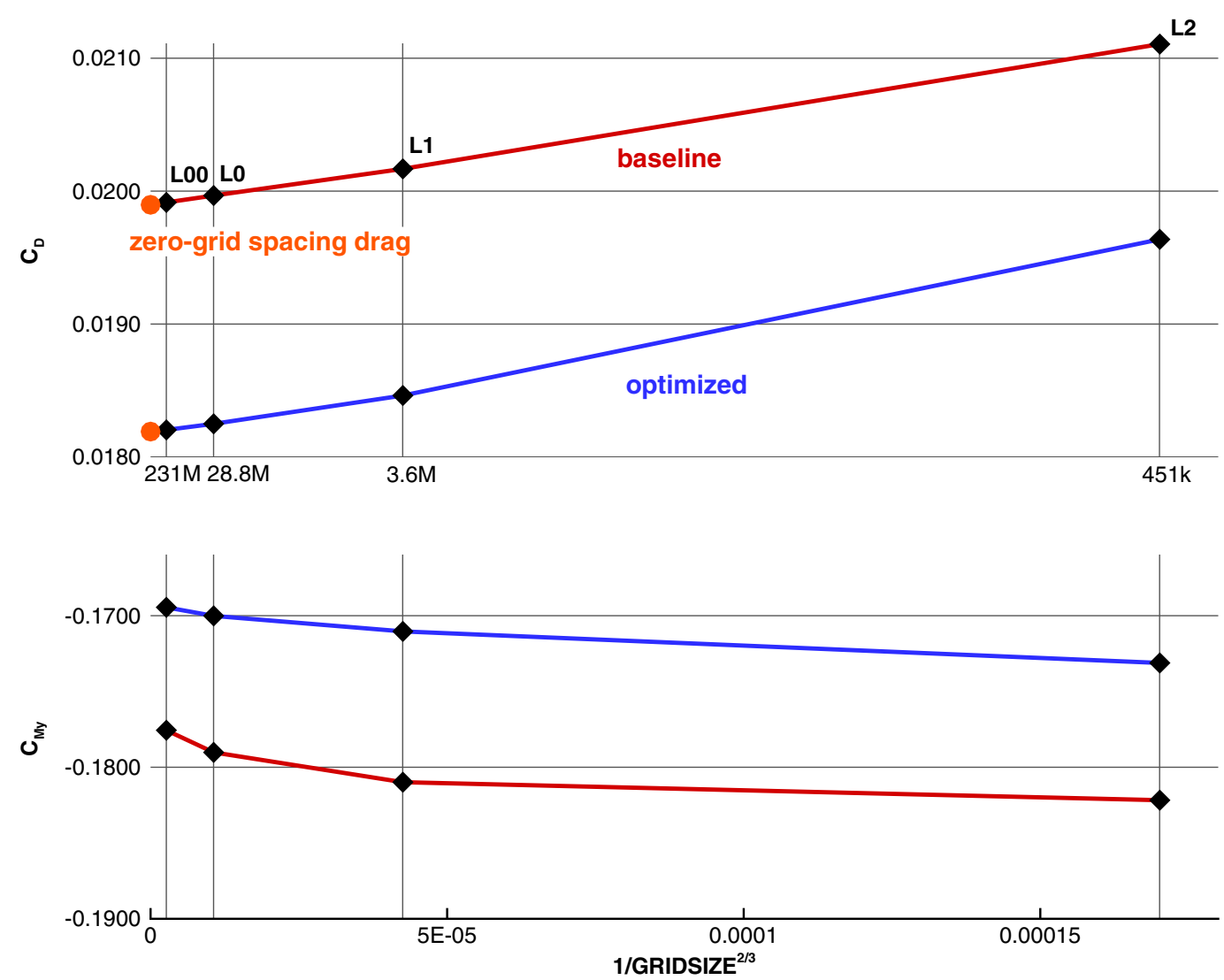

Fig. 6 The mesh-convergence study shows that the difference between the drag value computed with the 28.8 million grid and the zero-grid spacing drag is within one count.

constrained to be greater than or equal to the baseline volume, and so the optimizer drastically decreases the thickness of the airfoils on the outboard of the wing to the lower bounds, where there is less volume to be gained, while increasing the thickness near the root (up to 20\%), where the chords are larger and the volume-drag tradeoff is more favorable. Telidetzki et al. [24] observed similar trends in their results. The low outboard thickness would, in practice, incur a large structural weight penalty, and to trade off the reduction in drag and
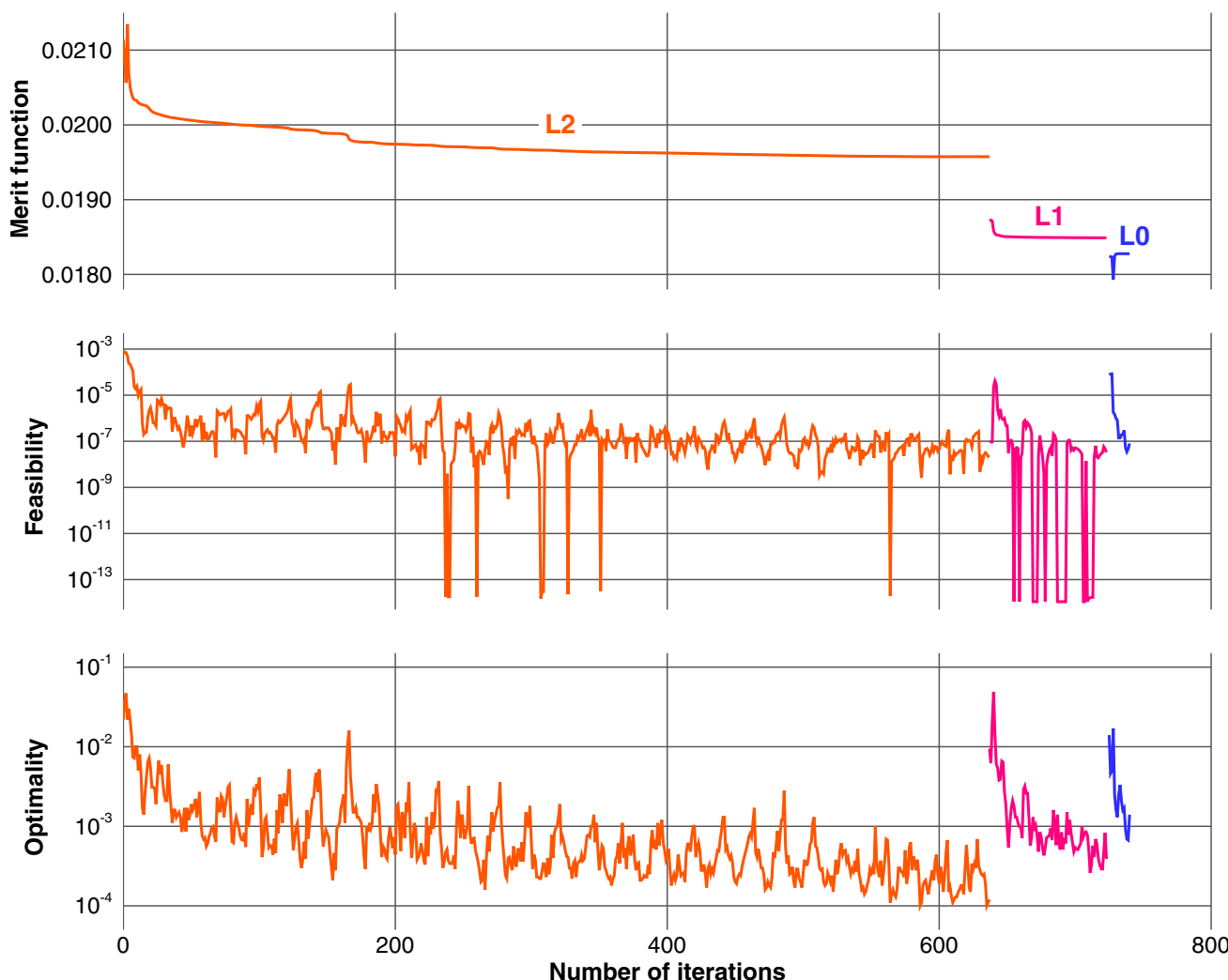

Fig. 7 Most of the computations are performed on the coarse grid. 


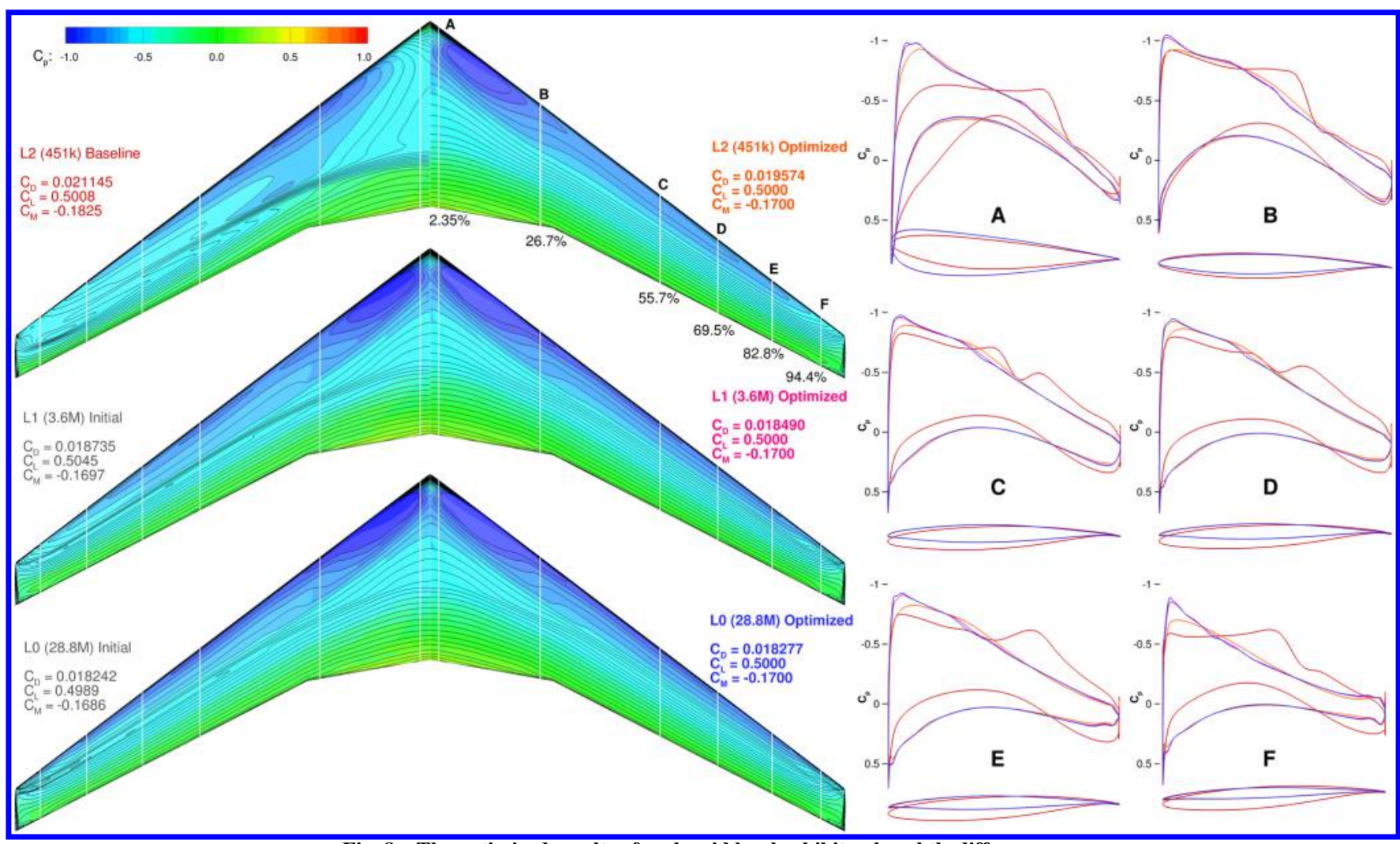

Fig. 8 The optimized results of each grid level exhibit only subtle differences.

increase in weight would require aerostructural optimization [32]. To obtain a more realistic design without resorting to aerostructural optimization, in Sec. VIII, we solve an additional optimization problem with a stricter thickness constraint.

The baseline wing exhibits a front of closely spaced pressure contour lines spanning a significant portion of the wing, indicating a shock. The optimized wing shows parallel pressure contour lines with uniform spacing, indicating a shock-free solution under the nominal flight condition. This is confirmed by the shock surface plots; we can see that the baseline wing has a shock on the upper surface, whereas the optimized wing does not show shocks under the design condition. The shock elimination can also be seen on the airfoil $C_{p}$ distributions. The sharp increase in local pressure due to the shock becomes a gradual change from the leading edge to the trailing edge.

Another noticeable feature in the optimized wing is the sharp leading edges in the outboard wing sections. The optimizer exploits a weakness in the problem formulation; with a single-point optimization, there is no penalty for thinning out the leading edge. In practice, however, sharp leading-edge airfoils experience adverse performance under offdesign conditions, because the flow is prone to separation at off-design angles of attack. We address these issues in more detail by performing a multipoint optimization in Sec. IX.

To ensure that the result of our single-point optimization has sufficient accuracy, we conducted a grid-convergence study of the optimized design. Table $\underline{3}$ summarizes the results for each grid level. The mesh-convergence plot for both the baseline and optimized geometry meshes is shown in Fig. 6. The zero-grid spacing drag, which was obtained using Richardson's extrapolation, is also plotted in the figure. We can see that the L0 mesh has sufficient accuracy; the

Table 4 The number of iterations on the LO grid is reduced to 18

\begin{tabular}{lcrcc}
\hline \hline Grid level & Iterations & Procs & Time, $h$ & Total proc-hr \\
\hline L2 & 638 & 64 & 29.3 & $1,875.2$ \\
L1 & 89 & 256 & 20.2 & $5,171.2$ \\
L0 & 18 & 1,248 & 11.1 & $13,852.8$ \\
\hline \hline
\end{tabular}

difference in the drag coefficient for the L0 mesh and the value obtained for the zero-grid spacing is within one drag count. The variation in drag coefficient between the baseline and optimized meshes is nearly constant for each grid level, which gives us confidence that the optimization using the coarse meshes represent the design space trends sufficiently well. Therefore, we perform the remaining optimization studies on the coarser mesh (L2), assuming that we capture the correct design trends.

\section{Multilevel Optimization Acceleration Technique}

In this section, we present an acceleration technique that reduced the overall computational cost of the optimization. Aerodynamic shape optimization is a computational intensive endeavor, where the majority of the computational effort is spent in the flow solutions and gradient evaluations. Therefore, many CFD researchers have tried to develop more efficient flow and adjoint solvers. Commonly used methods, such as multigrid, preconditioning, and variations on Newton-type methods, can improve the convergence of the solver, thus reducing the overall optimization time. Our flow solver has been significantly improved over the years to provide efficient and reliable flow solutions. Another area of improvement is the efficiency of the gradient computation. As mentioned in Sec. I, the adjoint method efficiently computes gradients with respect to large numbers of shape design variables. For our adjoint implementation, the cost of computing the gradient of a single function of interest with respect to hundreds or even thousands of shape design variables is lower than the cost of one flow solution [13].

Because we have improved the efficiency of our flow and adjoint solvers significantly over the last few years $[13,31,36]$, we seek new methods to further reduce the computational cost of the aerodynamic shape optimization. In this paper, we present a method that is inspired by the grid-sequencing procedure in CFD. Because it is less costly to compute both the flow solution and the gradient on a coarser grid, we perform the optimization first on the coarsest grid until a certain level of optimality is achieved. Then, we move to the next grid level and start with the optimal design variables from the coarser grid. Because the drag and lift coefficients are generally different for each grid level, the approximate Hessian (used by the gradient-based optimizer) must be restarted. We 


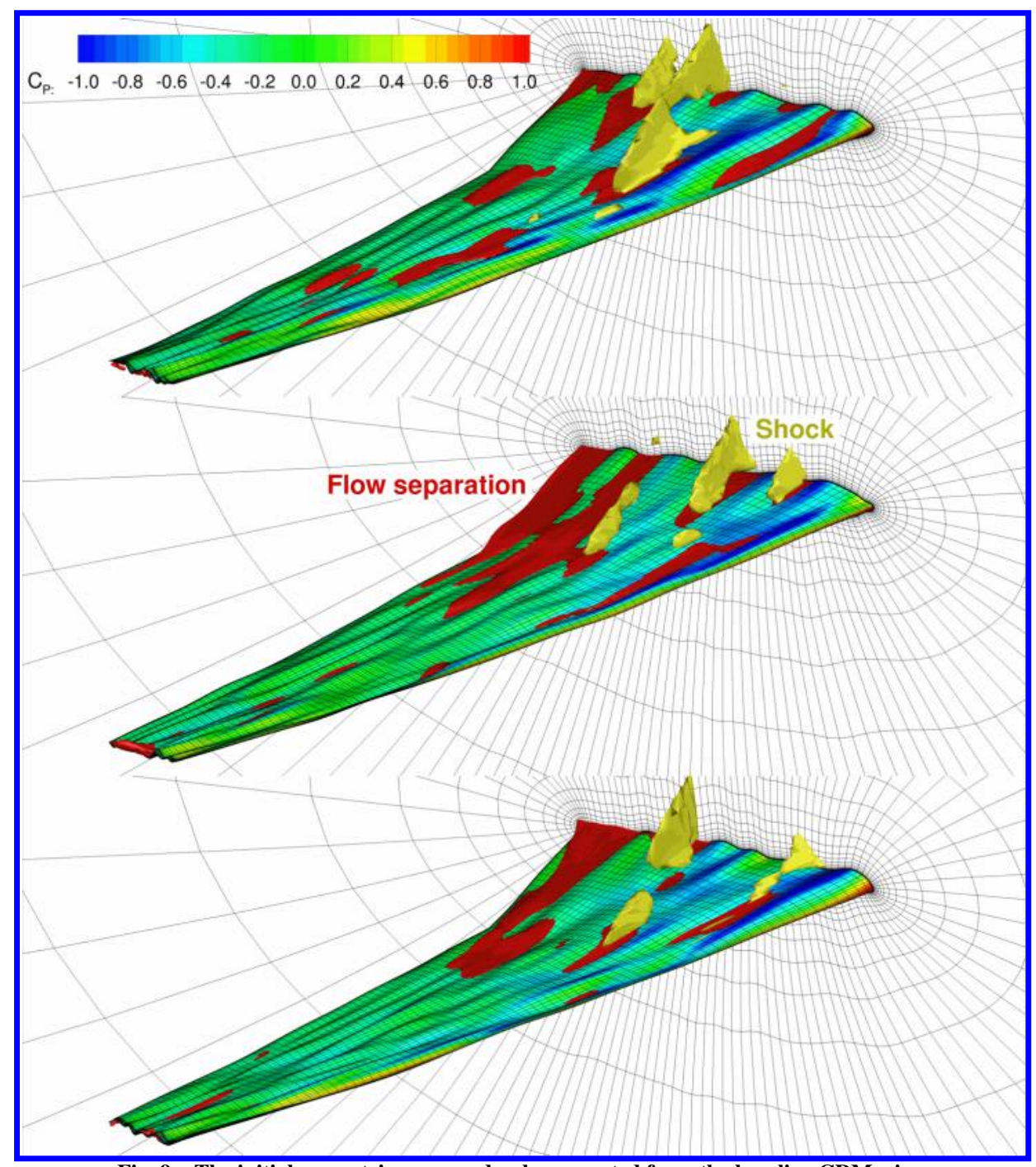

Fig. 9 The initial geometries are randomly generated from the baseline CRM wing.

repeat this process until the optimization on the finest grid has converged. Note that this procedure is different from traditional multigrid methods, where the coarse levels are revisited via multigrid cycles.

We used this procedure to obtain the optimal wing presented in the previous section. We use three grid levels: L2 (451,000 cells), L1 (3.6 million cells), and L0 (28.8 million cells). The merit function, optimality, and feasibility histories are plotted in Fig. 7; detailed definitions of these values can be found in the SNOPT manual [48]. We can see that most of the optimization iterations are performed on the coarse grid, and as a result, the number of the function and gradient evaluations on the successively finer grids is greatly reduced. Table 4 summarizes the computational time spent on each grid level. Thanks to the optimization with the coarser grids, only 18 iterations are needed on the $\mathrm{L} 0$ grid to converge the optimization. However, the L0 grid requires the largest computational effort, due to the high cost of the flow and adjoint solutions on this fine grid. Given that the cost per optimization iteration in the $\mathrm{L} 0$ grid is 770 processorhours (proc-hr) (compared to 2.9 proc-hr for the L2 grid), it is not feasible to perform an optimization using only the L0 grid. Assuming that the same number of iterations used for the L2 grid (638) would be needed for the $\mathrm{L} 0$ grid, the computational cost would be 23 times higher than that of the multilevel approach, which would correspond to 16 days using 1248 processors.

Figure 8 shows the initial and optimized results at each grid level. If we examine the results more closely, we see that the optimized results for the L2, L1, and L0 grids are all similar. This validates the underlying assumption of this method, that a coarser grid provides a good approximation to the design space of the finer grid when the set of design variables remains the same. Most of the computational effort on the subsequent grid levels is spent on smoothing out the shock that reappeared because of the finer grid spacing. This multilevel acceleration technique proved to significantly reduce the number of iterations needed to optimize in the fine grid, and the total computational effort was greatly reduced.

\section{Aerodynamic Shape Optimization Starting from a Random Geometry}

The existence of multiple local minima in RANS-based threedimensional aerodynamic shape optimization with respect to large numbers of design variables has yet to be explored. The problem is that, because of the high number of dimensions, the design space is difficult to visualize. In addition, the function evaluations are costly, making it challenging to explore the design space thoroughly and come to definitive conclusions.

We explore the multimodality of the single-point aerodynamic shape optimization problem described in Sec. IV, by solving separate optimizations starting from four different geometries. The first starting geometry is the CRM wing of Sec. IV. The other three starting geometries are randomly generated by applying a random perturbation to each design variable of the CRM wing, resulting in completely different geometries. The volume constraint is imposed, such that the volume of the baseline CRM wing is preserved. The initial starting points for the three random runs are shown in Fig. 9 . 


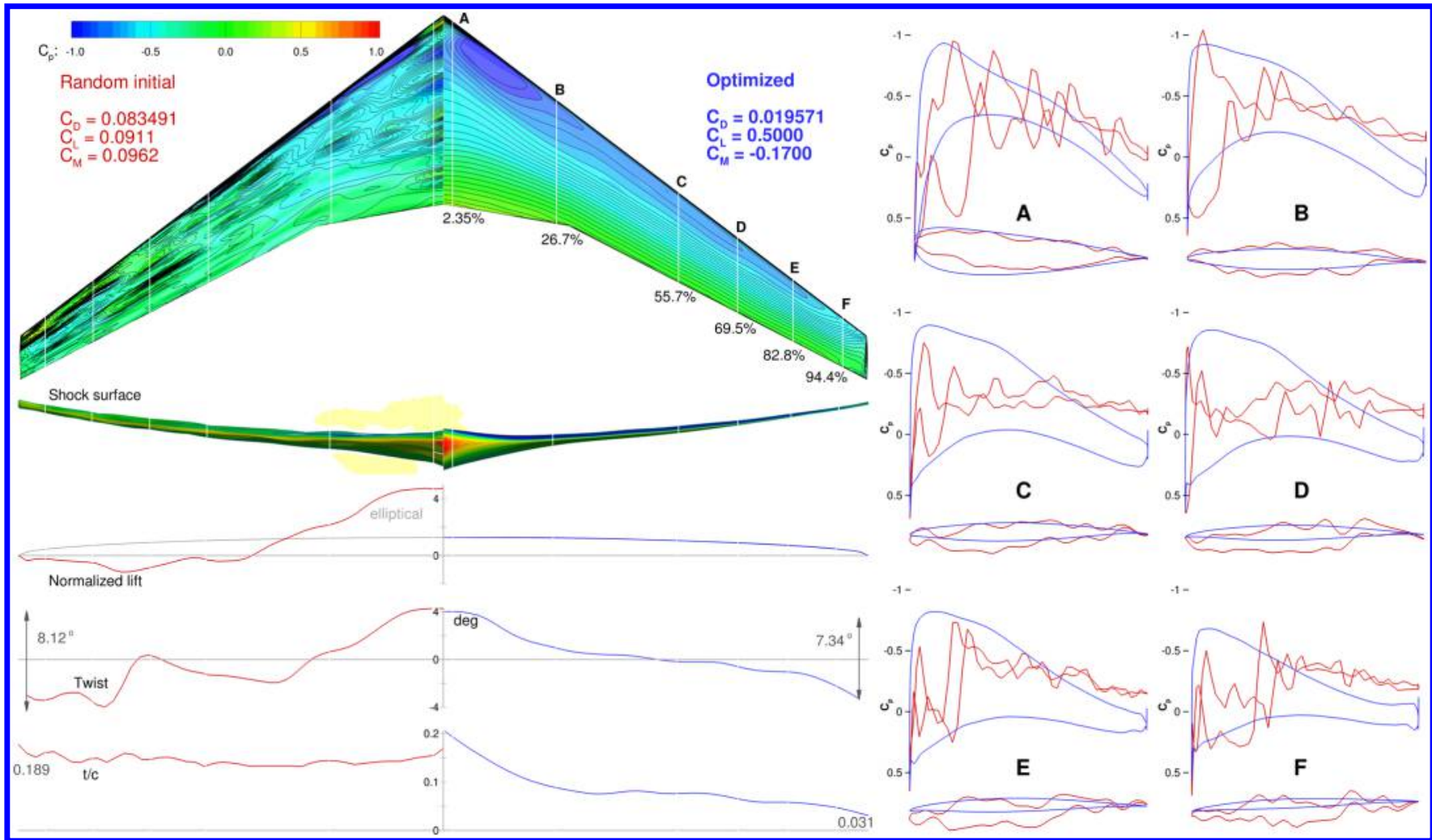

Fig. 10 The optimization manages to start from a random geometry and converge to an optimal wing that is shock-free.

The $C_{p}$ distribution is shown on the surface, along with a visualization of the shock and separation.

Figure 10 shows the optimized results from a random initial geometry. The optimization is performed on the L 2 grid. We can see that the performance of the initial design is extremely poor. This is no surprise because the airfoil shapes are unlike anything one would design; they exhibit oscillations and sharp edges, resulting in a wildly varying $C_{p}$ distribution. In addition, the flow solution is probably not accurate. In spite of these wild shapes and the inaccuracy of the flow solution, the gradients seem to point in the right direction because the

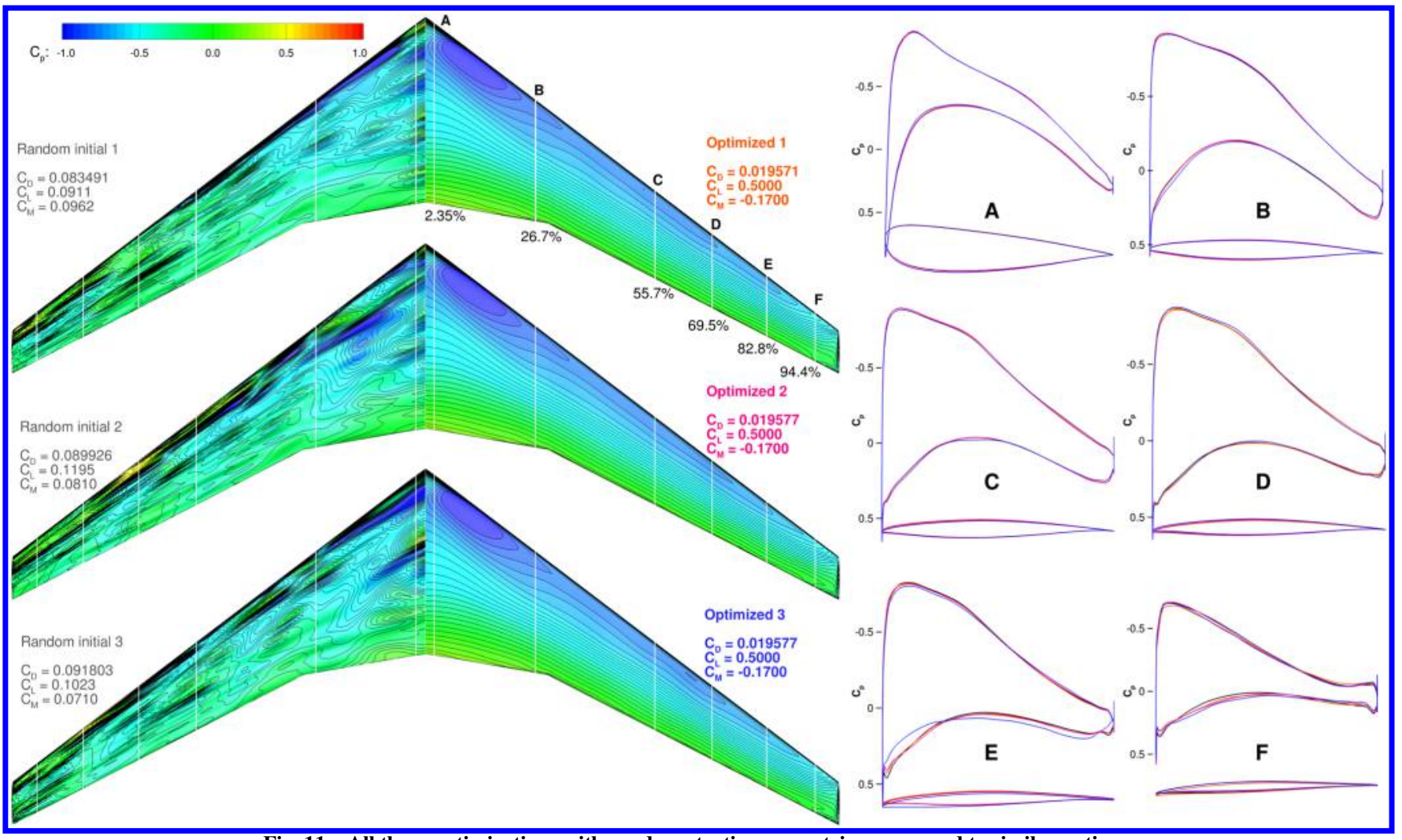

Fig. 11 All three optimizations with random starting geometries converged to similar optima. 


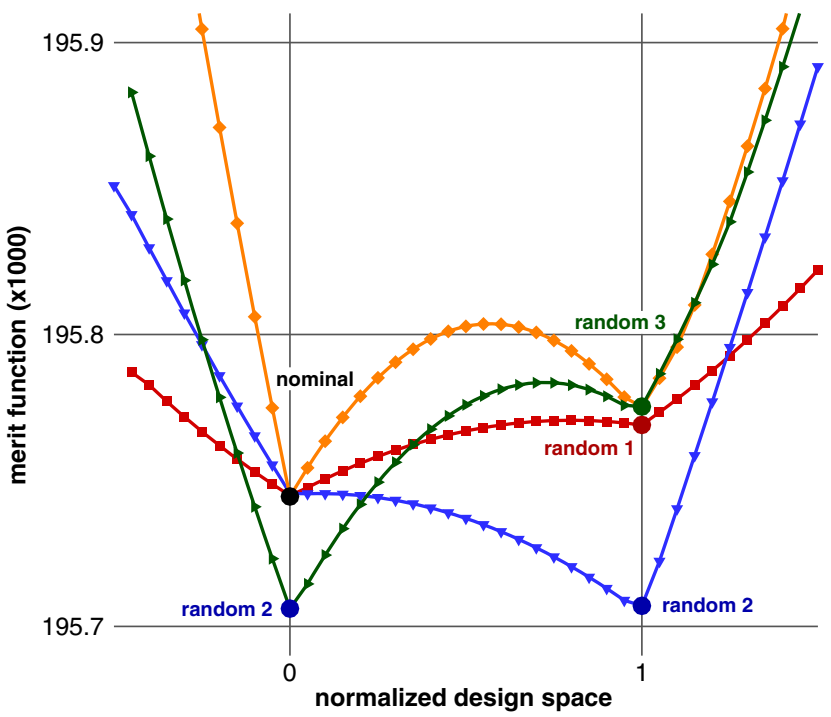

Fig. 12 The merit function values between optimized designs show the local minima.

optimizer is able to smooth out the airfoils and achieve a shock-free wing similar to the original single-point design presented in Sec. IV. All the constraints are met, and the lift distribution is close to elliptical. This optimization demonstrates the robustness of our aerodynamic optimization approach and showcases the power of the adjoint method. A movie of the optimization iteration history for this case is available in Supplemental Data S10.

We performed the same optimization for three random starting points and compared the results against each other, as well as against the single-point optimized wing, as shown in Fig. 11. The airfoil $C_{p}$ distributions of each optimized result and the nominal optimized result from Sec. IV are shown. Overall, there are only small differences between the four designs, as evidenced by the similar $C_{p}$ distributions and cross-sectional shapes. The difference in drag between all four designs is within one drag count. However, there are still some small visible differences, indicating the possibility that the design space is multimodal.

To further visualize this design space, we compute the merit function in the design space between two optimized designs, as shown in Fig. 12. The merit function is a combination of the objective function and the constraints [48]. We are able to visualize a slice of the design space by plotting the merit function along a line between two optima. A series of wing shapes are generated by linearly varying all

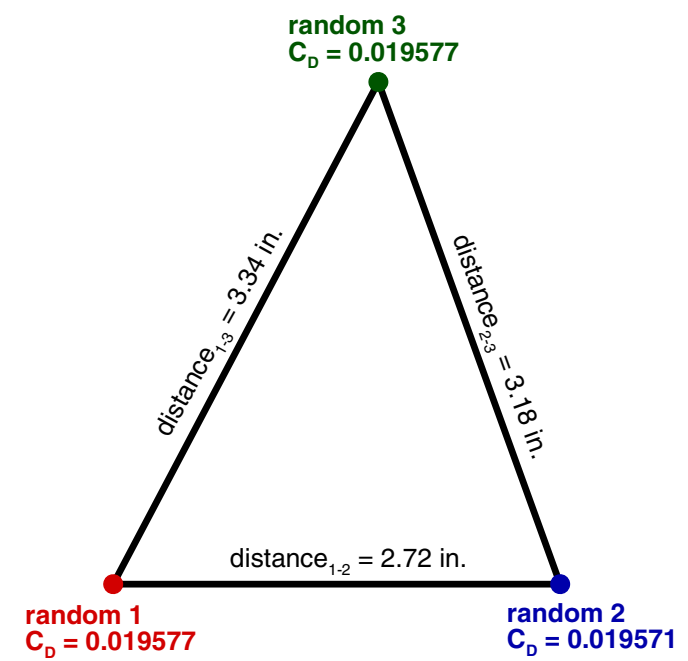

Fig. 13 The Euclidean distances between the multiple local minima are similar and are all under $3.4 \mathrm{in}$. (1.2\% of the mean aerodynamic chord). of the design variables. A CFD solution is solved for each of those designs to obtain the merit function. As shown in this figure, the merit function does appear to have multiple local minima, even though the values of the merit function are within one count among those optima. In addition, we also computed the mean difference between the design variables of each of the optimized designs as shown in Fig. 13. The three optima appear to be nearly equally spaced in the design space, with a Euclidean distance ranging from 2.72 to $3.34 \mathrm{in}$., which corresponds to only $1.2 \%$ of the mean aerodynamic chord. Based on this data, we believe that the design space for this aerodynamic shape optimization problem is mostly convex but that it has a small flat region that is multimodal. The humps and local minima could also be caused by the constraints.

\section{Effect of the Number of Shape Design Variables}

The cost of computing gradients with an efficient adjoint implementation is nearly independent of the number of design variables. We took advantage of this efficiency by optimizing with respect to 720 shape design variables in the previous sections. However, we would like to determine the tradeoff between the number of design variables and the optimal drag and to examine the effect on the computational cost of the optimization. Thus, in this section, we examine the effect of reducing the number of design variables. A series of new enlarged FFDs are created to ensure proper geometry embedding for small numbers of design variables. The shape design variables are distributed in a regular grid, where the finest grid has $15 \times 48=720$ design variables. The 15 chordwise stations correspond to 15 distinct airfoil shapes, while the shape of each airfoil is defined by 48 control points (half of these on the top, and the other half on the bottom).

We solve the optimization problem of Sec. IV using the L2 grid with variations in the number of defining airfoils and the number of points per airfoil. Figure 14 shows the resulting optimized designs for different numbers of airfoil control points and a fixed number of defining airfoils. Reducing the airfoil control points from 48 to 24 has a negligible effect on the optimal shape and pressure distribution, and the optimum drag increases by only 0.1 counts. As we further reduce the number of airfoil points to 12 and 6 , both the drag and pressure distribution show noticeable differences.

Variation in the number of defining airfoils follows a similar trend to the variation in the number of airfoil control points, as shown in Fig. 15. However, the drag penalty due to the number of airfoils is less severe than the penalty observed in the airfoil point reduction. Therefore, increasing the number of design variables in the chordwise direction is more beneficial than increasing the number of defining airfoils in the spanwise direction.

We also perform the optimization with a reduced number of shape design variables in both the chordwise and spanwise directions simultaneously, as shown in Fig. 16. From this study, we conclude that an adequate optimized design can be achieved with a smaller number of design variables; with $8 \times 24=192$ shape variables, the difference in the optimal drag coefficient is only 0.6 counts. Any further reduction in the number of design variables has a much larger impact on the optimal drag.

Figure 17 plots the convergence history for each optimization case. When we decrease the number of airfoil control points, the number of optimization iterations required decreases drastically. However, the number of defining airfoils has little effect on the optimization effort. This is in part because the adjoint computational cost is independent of the number of design variables. In addition, the coupled effects between design variables are much stronger between variables within an airfoil than between variables in different airfoils.

For an optimization process in which the computational cost scales with the number of design variables, such as when the gradients are computed via finite differences, or for gradient-free optimizers, a smaller number of design variables would significantly impact the optimized design. For example, for $3 \times 6=18$ variables, the drag of the optimized design would increase by 5.4 counts. 


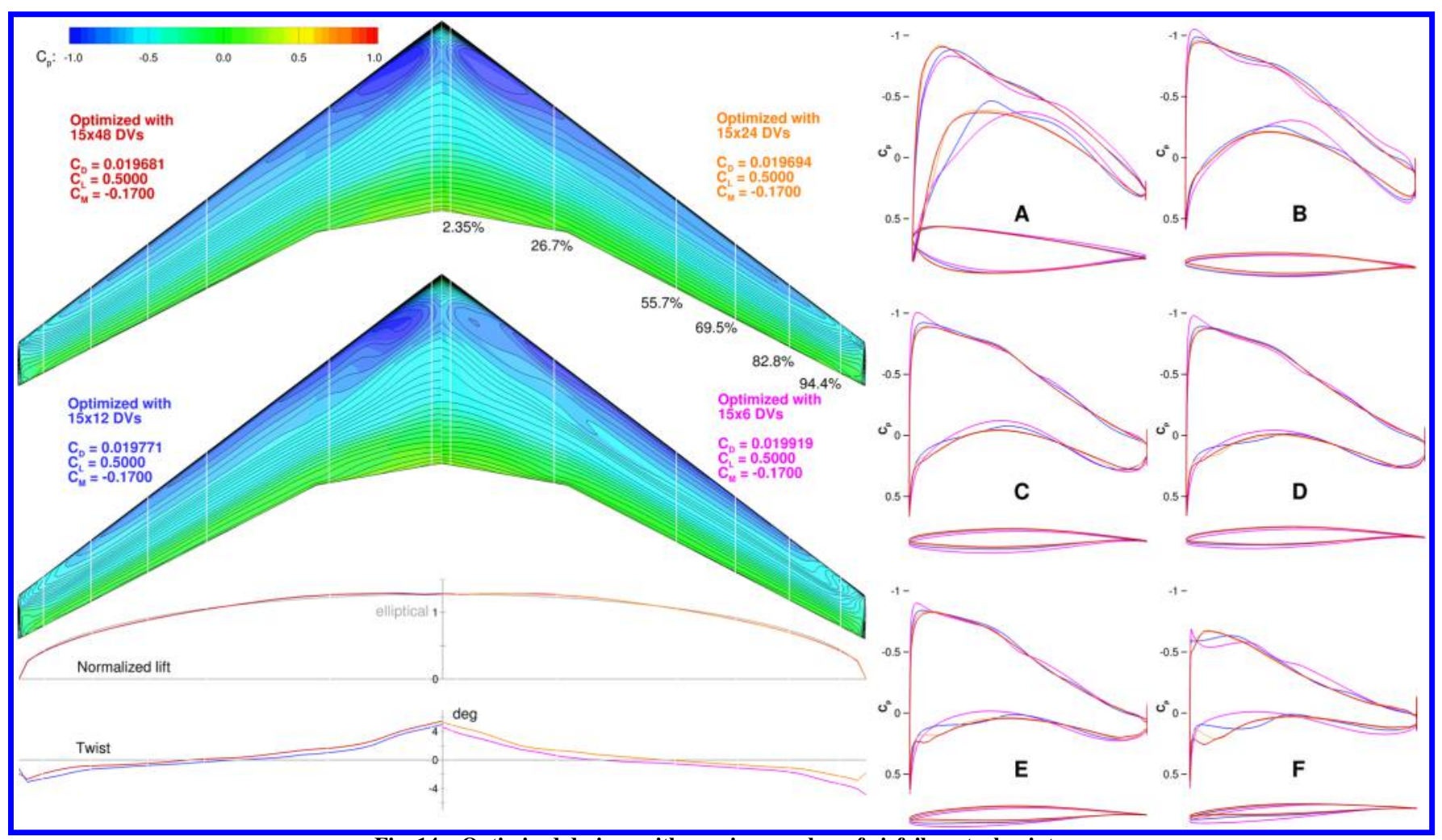

Fig. 14 Optimized designs with varying number of airfoil control points.

\section{Aerodynamic Shape Optimization Without Thickness Reduction}

As seen in Sec. IV, the optimized wing has a thickened root airfoil and an unrealistically thin tip airfoil. To address this issue, we solved an optimization problem identical to that solved in Sec. IV except for modified thickness constraints: all thicknesses must be greater than or equal to the baseline thickness (instead of being allowed to decrease to $25 \%$ of the baseline thickness). The optimization is performed on the L2 grid, and the results are shown in Fig. 18. A movie of the optimization iteration history for this case is available in Supplemental Data S11.

The results of the optimization with no thickness reduction are shown in black. The spanwise lift and twist distributions for the two cases are similar. However, the pressure distribution and airfoil

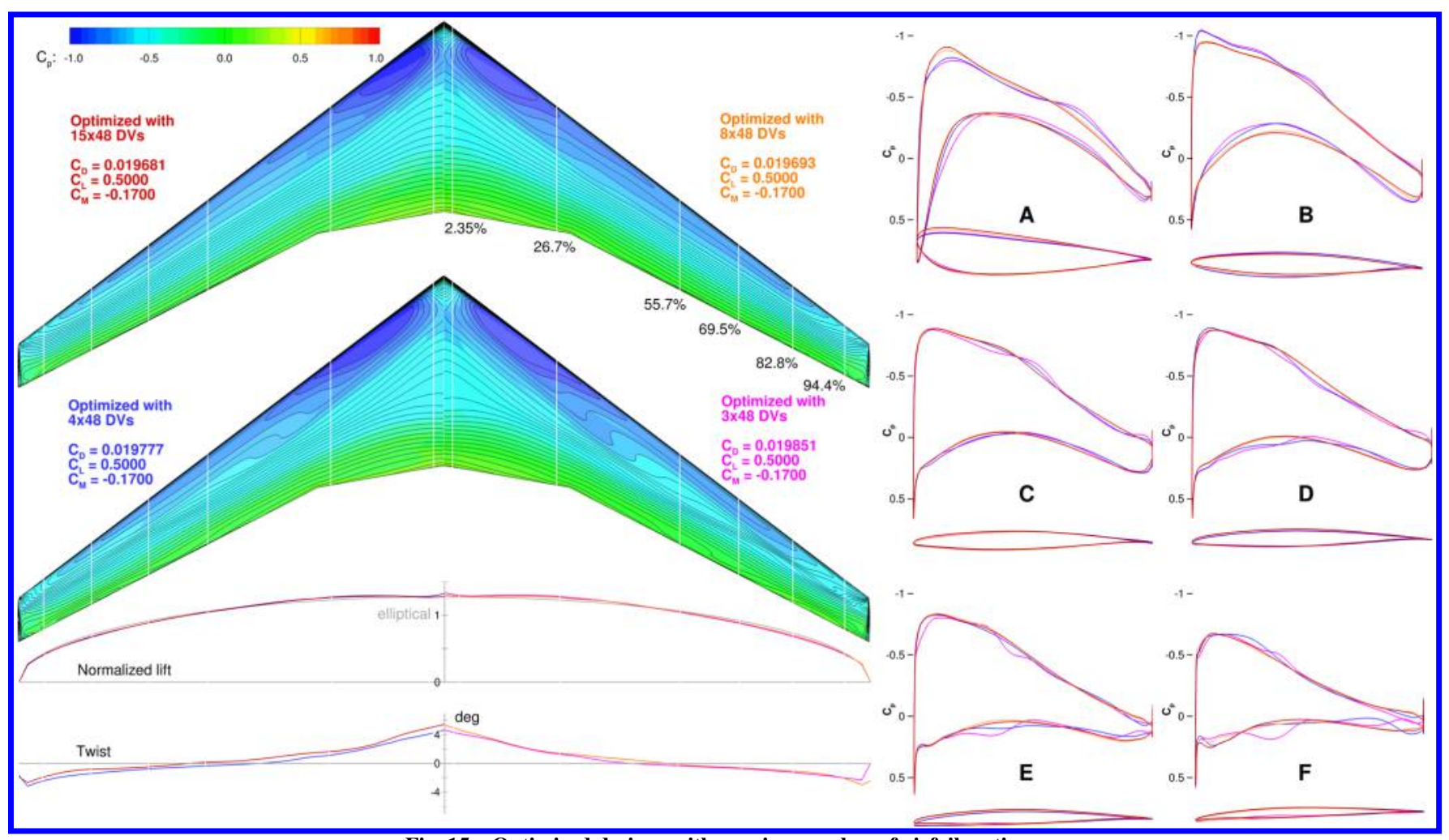




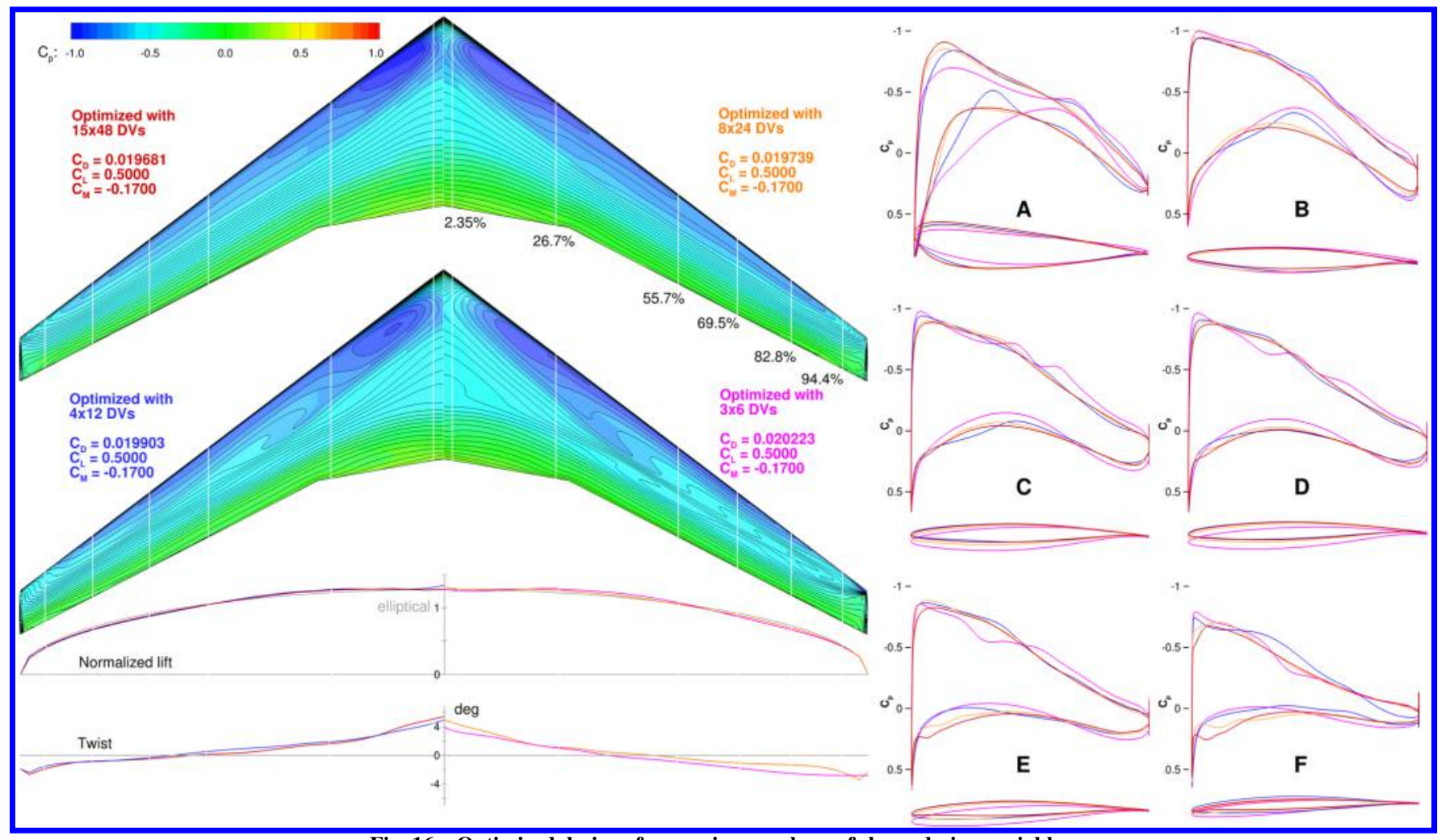

Fig. 16 Optimized designs for varying numbers of shape design variables.

shapes are significantly different, especially those near the wing root and wing tip. The mean difference between the baseline and optimized designs is only $1.1 \mathrm{in}$. The optimized wing with no thickness reduction has five additional drag counts when compared with the optimized wing that allowed $25 \%$ of the baseline thickness. This aerodynamic performance penalty may be compensated for by the reduction in the wing weight when structural design is considered. A detailed aerostructural optimization would be necessary to
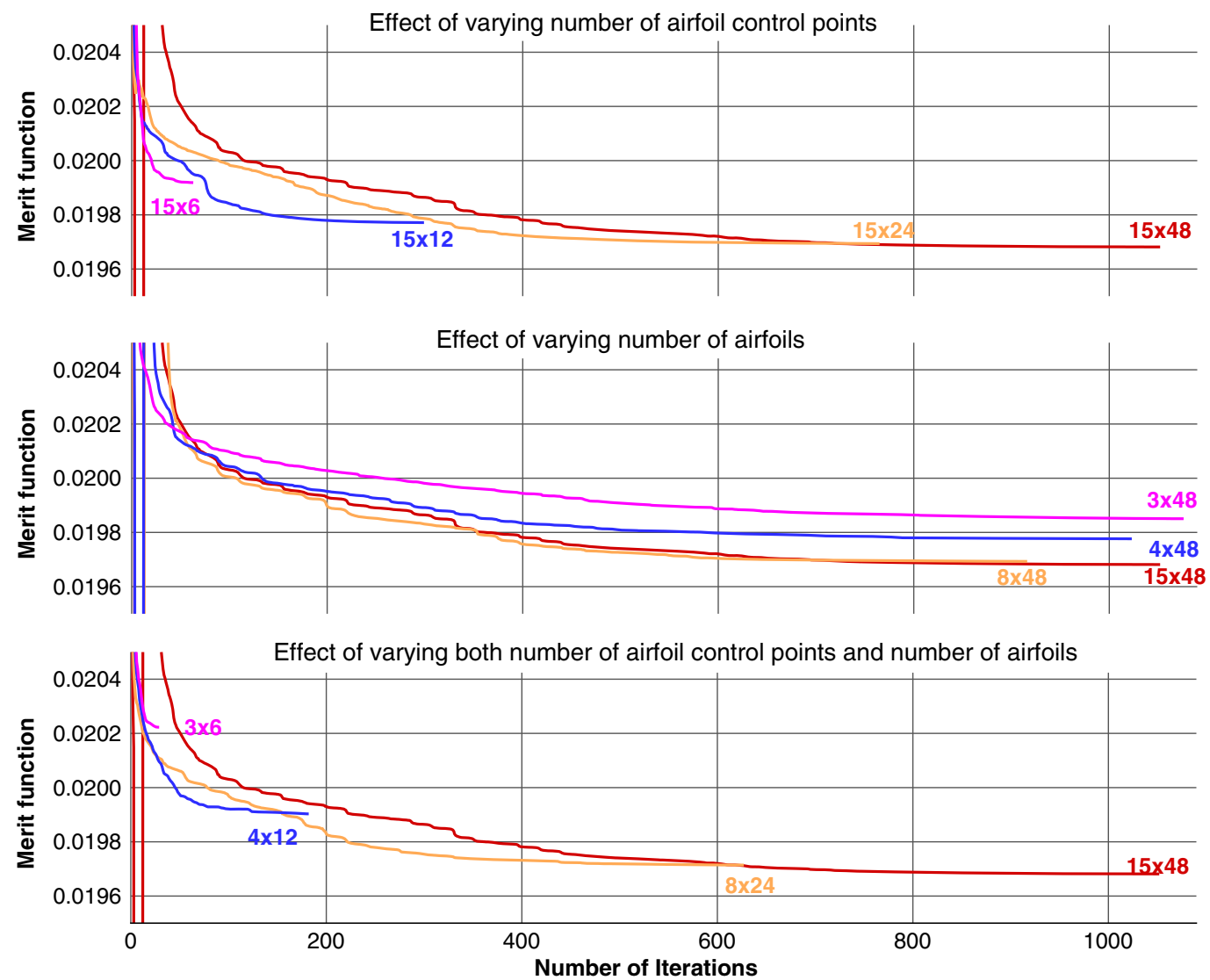

Fig. 17 The number of optimization iterations does not decrease significantly as the number of defining airfoils is decreased. 


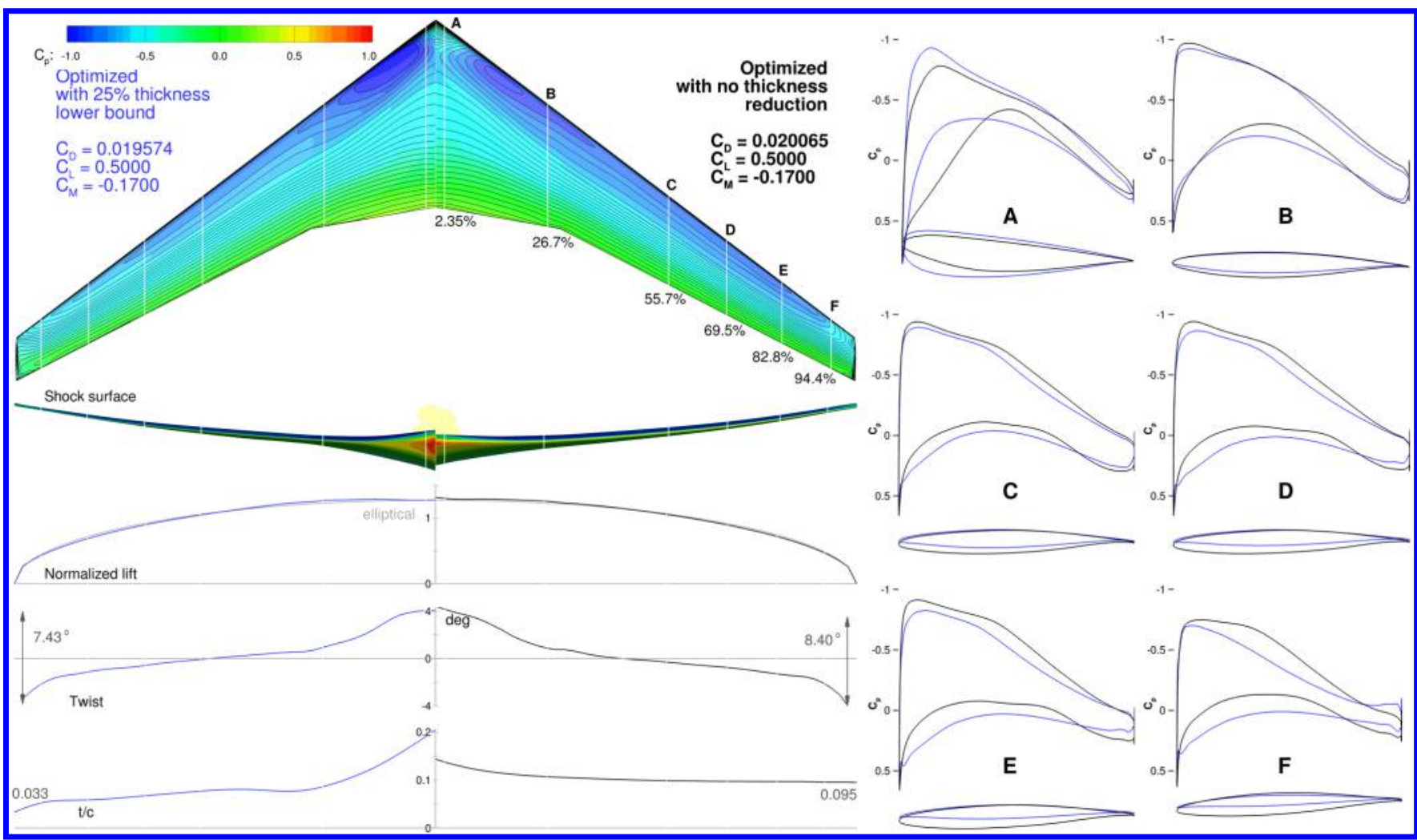

Fig. 18 The drag on the optimized wing is five counts higher if no airfoil thickness reduction is allowed.

examine the multidisciplinary tradeoffs involved [31,32]. In addition, the optimization takes significantly fewer iterations (296 iterations) as compared to the optimization in Sec. IV (638 iterations). This is due to the absence of volume-thickness trade resulting from the tighter thickness constraints.

\section{Multipoint Aerodynamic Shape Optimization}

Transport aircraft operate at multiple cruise conditions because of variability in the flight missions and air traffic control restrictions. Single-point optimization under the nominal cruise condition could overstate the benefit of the optimization because the optimization improves the on-design performance to the detriment of the offdesign performance. In Sec. IV, the single-point optimized wing exhibited an unrealistically sharp leading edge in the outboard of the wing. This was caused by a combination of the low value for the thickness constraints (25\% of the baseline) and the single-point formulation.

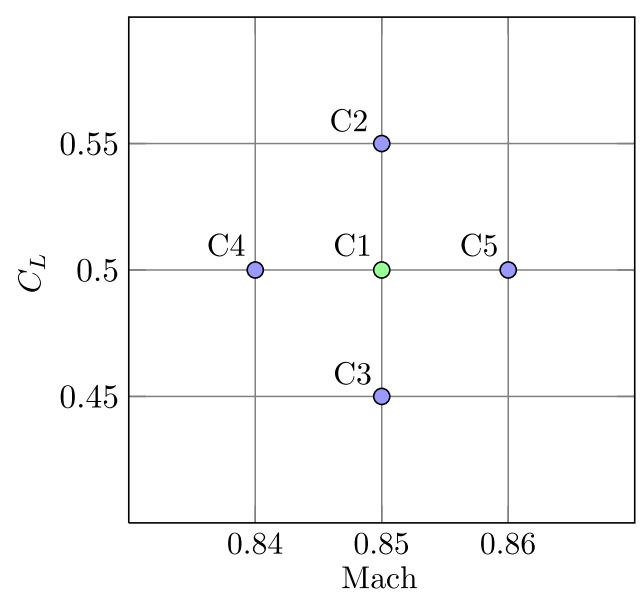

Fig. 19 The multipoint optimization flight conditions represent a fivepoint stencil in Mach- $C_{L}$ space.
A sharp leading edge is undesirable because it is prone to flow separation under off-design conditions. We address this issue by performing a multipoint optimization. The optimization is performed on the L2 grid. We choose five equally weighted flight conditions with different combinations of lift coefficient and the Mach number, as previously done by the authors [32]. The flight conditions are the nominal cruise, $\pm 10 \%$ of cruise $C_{L}$, and \pm 0.01 of cruise Mach, as shown in Fig. 19. More sophisticated ways of choosing multipoint flight conditions and their associated weights can be used, such as the automated procedure developed by Liem et al. [43] that minimizes fleet-level fuel burn. The objective function is the average drag coefficient for the five flight conditions, and the moment constraint is enforced only for the nominal flight condition.

A comparison of the single-point and multipoint optimized designs is shown in Fig. 20. A movie of the optimization iteration history for this case is available in Supplemental Data S12. The single-point results are shown on the left, and the multipoint results are shown on the right. The $C_{p}$ for the multipoint optimized result corresponds to the nominal condition. The multipoint sectional $C_{p}$ of flight conditions $2-5$ are plotted in gray. Unlike the shock-free design obtained with single-point optimization, the multipoint optimization settled on an optimal compromise between the flight conditions, resulting in a weak shock at all conditions. The leading edge is less sharp than that of the single-point optimized wing. Additional flight conditions, such as a low-speed flight condition, would be needed to further improve the leading edge. The overall pressure distribution of the multipoint design is similar to that of the single-point design. The twist and lift distributions are nearly identical. Most of the differences are in the chordwise $C_{p}$ distributions in the outer wing section. The drag coefficient under the nominal condition is approximately two counts higher. However, the performance under the off-design conditions is significantly improved. Similar trends were observed in the multipoint optimization of Vassberg and Jameson [23].

To demonstrate the robustness of the multipoint design, we plot $M L / D$ contours of the baseline, single-point, and multipoint designs with respect to $C_{L}$ and cruise Mach in Fig. 21. $M L / D$ provides a metric for quantifying aircraft range based on the Breguet range equation with constant thrust-specific fuel consumption. Although 


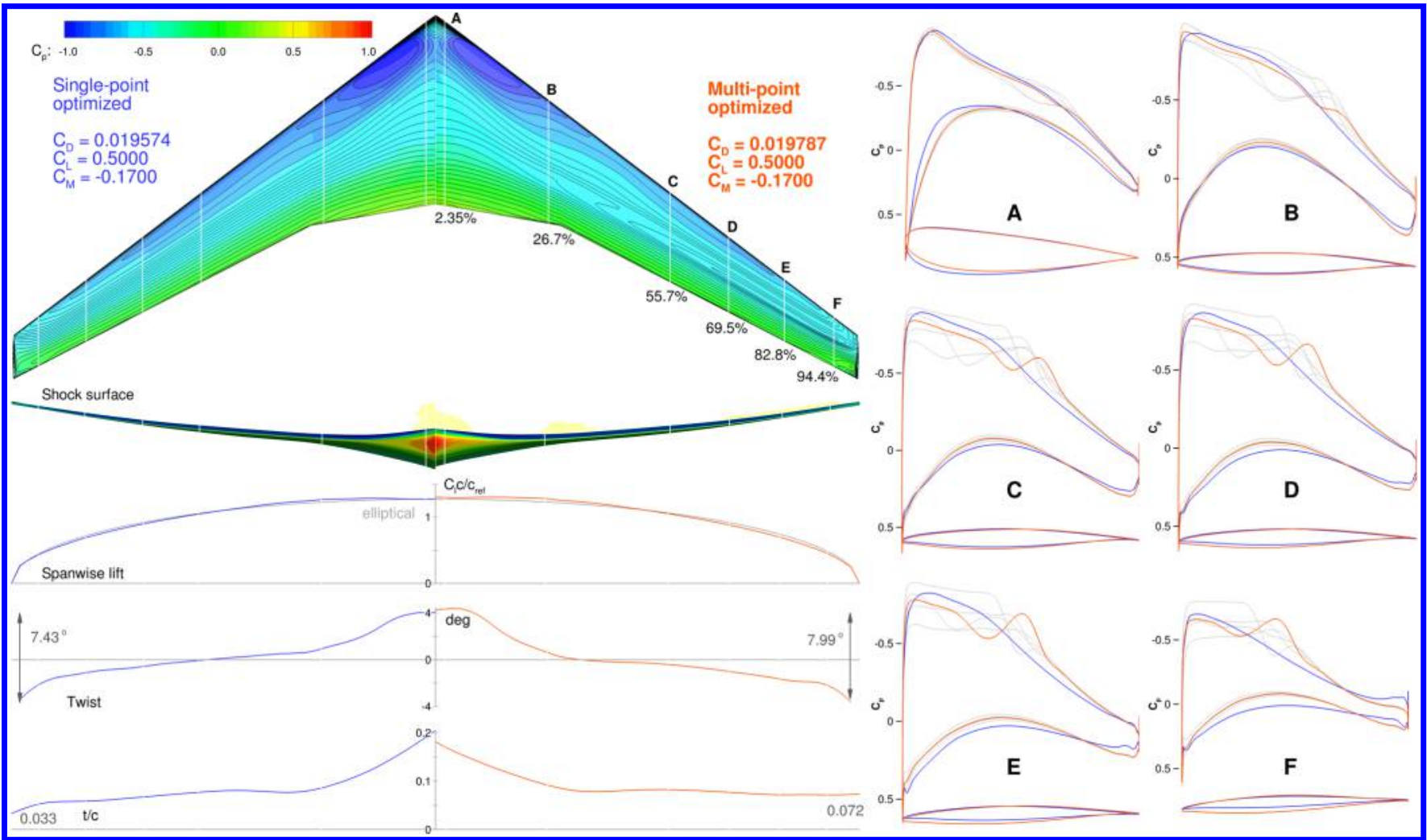

Fig. 20 The multipoint optimized wing has a weak shock on the upper surface for each flight condition.

the thrust-specific fuel consumption is actually not constant, assuming it to be constant is acceptable when comparing performance in a limited Mach-number range [49]. We add 100 drag counts to the computed drag to account for the drag due to the fuselage, tail, and nacelles, and we get more realistic $M L / D$ values.

The baseline maximum $M L / D$ is at a lower Mach number and a higher $C_{L}$ than that of the nominal flight condition. The single-point optimization increases the maximum $M L / D$ by $4 \%$ and moves this maximum toward the nominal cruise condition. If we examine the variation of $M L / D$ along the $C_{L}=0.5$ line, we see that the maximum occurs at the nominal Mach of 0.85 , which corresponds to a dip in a drag divergence plot.

For the multipoint optimization, the optimized flight conditions are distributed in the Mach- $C_{L}$ space, resulting in a flattened $M L / D$ variation near the maximum, which means that we have more uniform performance for a range of flight conditions. In aircraft design, the $99 \%$ value of the maximum $M L / D$ contour is often used to examine the robustness of the design [20]. The point with the highest Mach number on that contour line corresponds to the longrange cruise point, which is the point at which the aircraft can fly at a higher speed by incurring a $1 \%$ increase in fuel burn [50]. In this case, we see that the $99 \%$ value of the maximum $M L / D$ contour of the multipoint design is larger than that of the single-point optimum, indicating a more robust design.

The $M L / D_{\max }$ of the multipoint design is slightly higher than the maximum for the single-point design. Although this seems counterintuitive, it can be explained by the fact that the analysis conditions do not line up with the optimized maximum location. The optimizer has no information about the exact flight condition of the peak location, and thus it does not directly control the value at that location. A potential remedy for this mismatch would be the addition of two degrees of freedom to the optimization problem: the nominal Mach number and the nominal lift coefficient. This would allow the optimizer to track the $M L / D_{\max }$ location during the optimization; upon convergence, the objective value would reflect the maximum possible performance in the $M-C_{L}$ space. Performing such an optimization, however, would require propulsion and operating cost models and would involve multidisciplinary tradeoffs between aerodynamics and these other disciplines [43].

\section{Conclusions}

In this paper, we have presented an extensive study of the CRM wing shape optimization benchmark defined by the Aerodynamic Design Optimization Discussion Group (ADODG). Both the baseline and optimized geometries and meshes are available in Supplemental Data S1-S8. The drag coefficient is minimized for one flight condition with respect to 720 shape design variables, subject to lift, pitching moment, and geometric constraints, using grids with up to 28.8 million cells. The drag coefficient of the optimized design was reduced by $8.5 \%$ relative to the CRM baseline: from 199.7 to 182.8 counts, with a zero-grid spacing value of 181.9 counts. We implemented a multilevel optimization procedure that significantly reduced the total computational time.

The single-point optimized design exhibits a small thickness-tochord ratio $(3.3 \%)$ at the tip, which would incur a large structural weight penalty in a real wing. Thus, we performed an additional optimization that did not allow for thickness reduction. Although the optimal drag increased by five counts relative to the nominal case, the associated reduction in structural weight would likely offset this penalty in a real wing when considering the overall aircraft performance.

The multimodality of the aerodynamic shape optimization problem was examined by starting optimizations from randomly generated initial geometries. All optimal wings had similar airfoil shapes, with a mean difference of 1.2 in. The variation of the merit function between the multiple local optima confirm that these points are indeed local minima and indicate that the design space consists of a convex bowl with a small flat bottom that is multimodal. Based on our data, the minimum drag coefficient values were within 0.1 counts $(0.05 \%)$, and the radius of this flat bottom seems to be about 1.6 in. Given these small differences, it does not seem worthwhile to put much effort into finding the global minimum for this problem. 


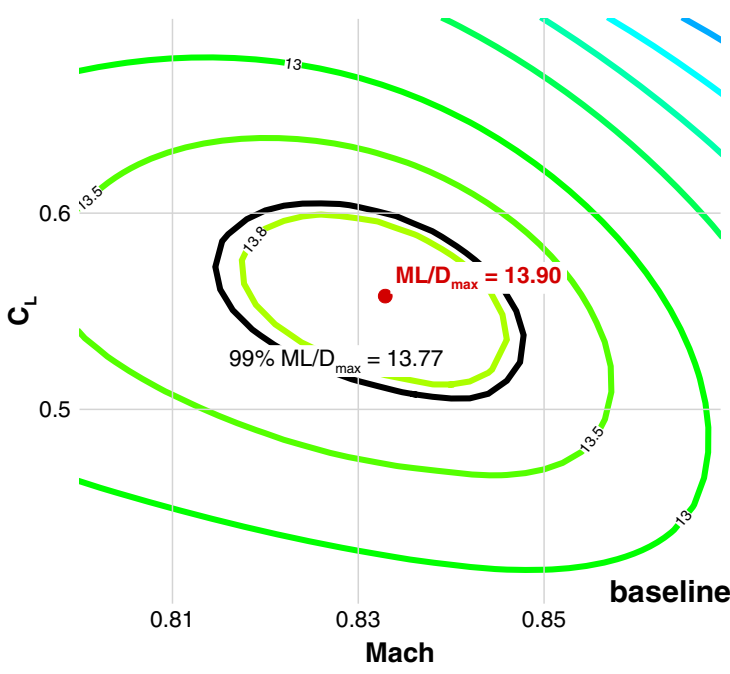

a) Baseline

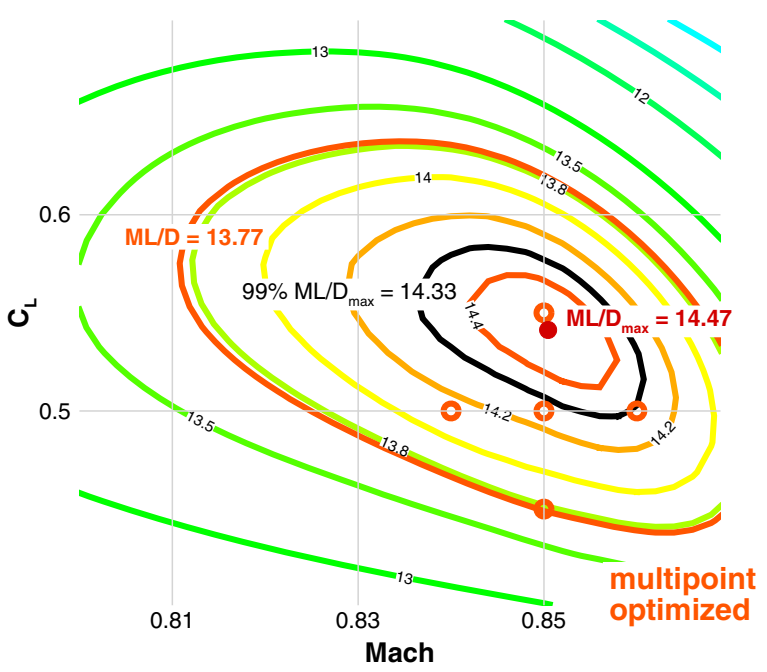

c) Multipoint optimized

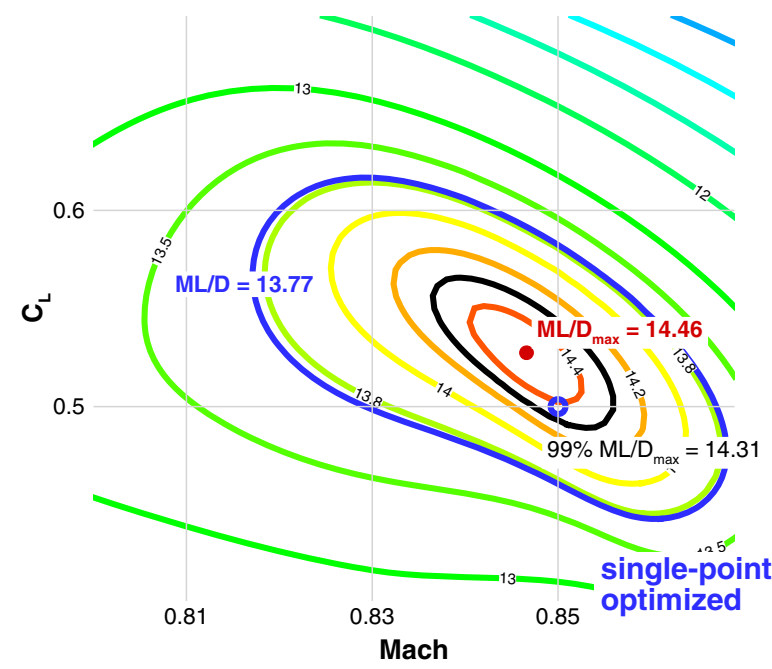

b) Single-point optimized

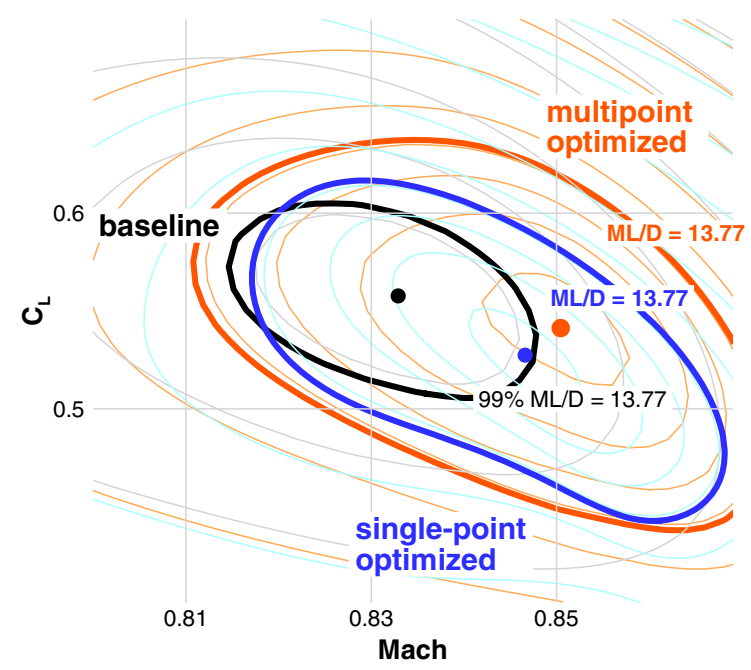

d) Comparison of baseline, single, and multipoint

Fig. 21 The multipoint optimized wing has better off-design performance and is more robust to changes in flight conditions.

We studied the effect of the design variables by varying the number of defining airfoil sections and the number of control points for each of those sections. Reducing the number of airfoil control points from 48 to 12 resulted in a 0.9 -count drag increase. The total number of optimization iterations also reduced with the number of airfoil control points. The number of airfoil sections has a similar influence on the optimized drag. However, decreasing the number of airfoil sections while keeping the number of airfoil control points constant did not affect the overall computational cost in a significant way. We found that the optimization with eight airfoil sections and 24 control points per section (192 design variables) provided the best tradeoff; it increased the optimal drag by only 0.6 counts relative to the 720 variable case (15 airfoils with 48 points each), while requiring $40 \%$ fewer optimization iterations.

Finally, we performed a multipoint optimization of the CRM wing. This resulted in a more robust design than that of the single-point optimization, as evidenced by the enlarged contour of the $99 \%$ maximum $M L / D$. We also compared the contours of $M L / D$ for the single-point baseline optimum and the multipoint optimum. Both the single-point and multipoint optimizations shifted the maximum $M L / D$ toward the nominal flight condition.

This CRM wing aerodynamic shape optimization problem is a valuable benchmark for the wing design optimization community, and we hope that more researchers tackle this problem. The ADODG is also expected to expand this suite of benchmark problems in the near future.
This aerodynamic design optimization problem is limited to a fixed-wing planform, but it is an excellent first step. To consider span and sweep, and to eliminate the explicit thickness constraints, it is necessary to consider the tradeoffs between drag and structural weight, which has been done in an optimal way using aerostructural optimization [32]. To take full advantage of the optimization, we should also include the flight conditions as design variables, but then we would have to solve an even more complex MDO problem that considers propulsion, mission analysis, and economics.

\section{Acknowledgments}

This research was partially funded by NASA under grant number NNX11AI19A. The computations were performed on the Flux HPC cluster at the University of Michigan Center of Advanced Computing and on the Gordon cluster of the Extreme Science and Engineering Discovery Environment, which is supported by National Science Foundation grant number ACI-1053575. The authors would like to thank S. Andrew Ning for his thorough review and suggestions, as well as our MDOlab colleagues for numerous ideas. Finally, the authors are thankful for the constructive feedback provided by the anonymous reviewers.

\section{References}

[1] Newton, S. I., Philosophice Naturalis Principia Mathematica, Imprimatur. s. Pepys Reg. Soc. Praeses., London, 1686. 
[2] Hicks, R. M., Murman, E. M., and Vanderplaats, G. N., "An Assessment of Airfoil Design by Numerical Optimization,” NASA TM-X-3092, 1974.

-[3] Hicks, R. M., and Henne, P. A., "Wing Design by Numerical Optimization," Journal of Aircraft, Vol. 15, No. 7, 1978, pp. 407-412. doi: $10.2514 / 3.58379$

[4] Peter, J. E. V., and Dwight, R. P., "Numerical Sensitivity Analysis for Aerodynamic Optimization: A Survey of Approaches," Computers and Fluids, Vol. 39, No. 3, 2010, pp. 373-391. doi:10.1016/j.compfluid.2009.09.013

-[5] Martins, J. R. R. A., and Hwang, J. T., "Review and Unification of Methods for Computing Derivatives of Multidisciplinary Computational Models," AIAA Journal, Vol. 51, No. 11, 2013, pp. 2582-2599. doi:10.2514/1.J052184

-[6] Pironneau, O., "On Optimum Profiles in Stokes Flow," Journal of Fluid Mechanics, Vol. 59, No. 1, 1973, pp. 117-128. doi:10.1017/S002211207300145X

-[7] Pironneau, O., "On Optimum Design in Fluid Mechanics," Journal of Fluid Mechanics, Vol. 64, No. 1, 1974, pp. 97-110. doi: $10.1017 /$ S0022112074002023

- [8] Jameson, A., "Aerodynamic Design via Control Theory," Journal of Scientific Computing, Vol. 3, No. 3, 1988, pp. 233-260. doi:10.1007/BF01061285

-[9] Reuther, J. J., Jameson, A., Alonso, J. J., Rimlinger, M. J., and Saunders, D., "Constrained Multipoint Aerodynamic Shape Optimization Using an Adjoint Formulation and Parallel Computers, Part 1," Journal of Aircraft, Vol. 36, No. 1, 1999, pp. 51-60. doi: $10.2514 / 2.2413$

[10] Reuther, J. J., Jameson, A., Alonso, J. J., Rimlinger, M. J., and Saunders, D., "Constrained Multipoint Aerodynamic Shape Optimization Using an Adjoint Formulation and Parallel Computers, Part 2," Journal of Aircraft, Vol. 36, No. 1, 1999, pp. 61-74. doi: $10.2514 / 2.2414$

[11] Hicken, J. E., and Zingg, D. W., "Aerodynamic Optimization Algorithm with Integrated Geometry Parameterization and Mesh Movement," AIAA Journal, Vol. 48, No. 2, 2010, pp. 400-413. doi: $10.2514 / 1.44033$

[12] Hicken, J. E., and Zingg, D. W., "Induced-Drag Minimization of Nonplanar Geometries Based on the Euler Equations," AIAA Journal, Vol. 48, No. 11, 2010, pp. 2564-2575. doi:10.2514/1.J050379

[13] Lyu, Z., Kenway, G., Paige, C., and Martins, J. R. R. A., "Automatic Differentiation Adjoint of the Reynolds-Averaged Navier-Stokes Equations with a Turbulence Model," 21st AIAA Computational Fluid Dynamics Conference, AIAA Paper 2013-2581, June 2013.

[14] Jameson, A., Martinelli, L., and Pierce, N., "Optimum Aerodynamic Design Using the Navier-Stokes Equations," Theoretical and Computational Fluid Dynamics, Vol. 10, Nos. 1-4, 1998, pp. 213-237. doi:10.1007/s001620050060

- [15] Anderson, W. K., and Bonhaus, D. L., "Airfoil Design on Unstructured Grids for Turbulent Flows," AIAA Journal, Vol. 37, No. 2, 1999, pp. $185-191$. doi: $10.2514 / 2.712$

[16] Nielsen, E. J., and Anderson, W. K., "Aerodynamic Design Optimization on Unstructured Meshes Using the Navier-Stokes Equations," AIAA Journal, Vol. 37, No. 11, 1999, pp. 1411-1419. doi: $10.2514 / 2.640$

[17] Dwight, R. P., and Brezillon, J., "Efficient and Robust Algorithms for Solution of the Adjoint Compressible Navier-Stokes Equations with Applications," International Journal for Numerical Methods in Fluids, Vol. 60, No. 4, 2009, pp. 365-389. doi:10.1002/fld.v60:4

- [18] Brezillon, J., and Dwight, R. P., "Applications of a Discrete Viscous Adjoint Method for Aerodynamic Shape Optimisation of 3D Configurations," CEAS Aeronautical Journal, Vol. 3, No. 1, 2012, pp. 25-34. doi:10.1007/s13272-011-0038-0

[19] Vassberg, J., "Introduction: Drag Prediction Workshop," Journal of Aircraft, Vol. 45, No. 3, 2008, p. 737. doi: $10.2514 / 1.37761$

[20] Vassberg, J., Dehaan, M., Rivers, M., and Wahls, R., "Development of a Common Research Model for Applied CFD Validation Studies," 26th AIAA Applied Aerodynamics Conference, AIAA Paper 2008-6919, Aug. 2008. doi: $10.2514 / 6.2008-6919$

[21] Vassberg, J., "A Unified Baseline Grid About the Common Research Model Wing/Body for the Fifth AIAA CFD Drag Prediction Workshop (Invited)," 29th AIAA Applied Aerodynamics Conference, AIAA Paper 2011-3508, July 2011. doi: $10.2514 / 6.2011-3508$
[22] Lyu, Z., Kenway, G. K. W., and Martins, J. R. R. A., "RANS-Based Aerodynamic Shape Optimization Investigations of the Common Research Model Wing," 52nd Aerospace Sciences Meeting, AIAA Paper 2014-0567, Jan. 2014.

[23] Vassberg, J., and Jameson, A., "Influence of Shape Parameterization on Aerodynamic Shape Optimization," Von Karman Inst., TR, VKI Lecture-III, Brussels, April 2014.

[24] Telidetzki, K., Osusky, L., and Zingg, D. W., "Application of Jetstream to a Suite of Aerodynamic Shape Optimization Problems," 52nd Aerospace Sciences Meeting, AIAA Paper 2014-0571, Feb. 2014. doi: $10.2514 / 6.2014-0571$

[25] Carrier, G., Destarac, D., Dumont, A., Meheut, M., Din, I. S. E., Peter, J., Khelil, S. B., Brezillon, J., and Pestana, M., "Gradient-Based Aerodynamic Optimization with the elsA Software," 52nd Aerospace Sciences Meeting, AIAA Paper 2014-0568, Feb. 2014. doi: $10.2514 / 6.2014-0568$

[26] Lyu, Z., and Martins, J. R. R. A., "RANS-Based Aerodynamic Shape Optimization of a Blended-Wing-Body Aircraft," 21st AIAA Computational Fluid Dynamics Conference, AIAA Paper 2013-2586, June 2013.

[27] Lyu, Z., and Martins, J. R. R. A., "Aerodynamic Design Optimization Studies of a Blended-Wing-Body Aircraft," Journal of Aircraft (accepted for publication) doi:10.2514/1.C032491

[28] Holst, T. L., and Pulliam, T. H., "Aerodynamic Shape Optimization Using a Real-Number-Encoded Genetic Algorithm," 19th AIAA Applied Aerodynamics Conference, AIAA Paper 2001-2473, 2001. doi:10.2514/6.2001-2473

[29] Sasaki, D., Morikawa, M., Obayashi, S., and Nakahashi, K., "Aerodynamic Shape Optimization of Supersonic Wings by Adaptive Range Multiobjective Genetic Algorithms," Evolutionary MultiCriterion Optimization, Lecture Notes in Computer Science, Vol. 1993, Springer, Berlin, 2001, pp. 639-652. doi:10.1007/3-540-44719-9_45

[30] Chernukhin, O., and Zingg, D. W., "Multimodality and Global Optimization in Aerodynamic Design," AIAA Journal, Vol. 51, No. 6, 2013, pp. 1342-1354. doi:10.2514/1.J051835

[31] Kenway, G. K. W., Kennedy, G. J., and Martins, J. R. R. A., "Scalable Parallel Approach for High-Fidelity Steady-State Aeroelastic Analysis and Adjoint Derivative Computations," AIAA Journal, Vol. 52, No. 5, 2014, pp. 935-951. doi:10.2514/1.J052255

[32] Kenway, G. K. W., and Martins, J. R. R. A., "Multipoint High-Fidelity Aerostructural Optimization of a Transport Aircraft Configuration," Journal of Aircraft, Vol. 51, No. 1, 2014, pp. 144-160. doi:10.2514/1.C032150

[33] Kenway, G. K., Kennedy, G. J., and Martins, J. R. R. A., "A CAD-Free Approach to High-Fidelity Aerostructural Optimization," 13th AIAA/ ISSMO Multidisciplinary Analysis Optimization Conference, AIAA Paper 2010-9231, 2010. doi: $10.2514 / 6.2010-9231$

[34] van der Weide, E., Kalitzin, G., Schluter, J., and Alonso, J., "Unsteady Turbomachinery Computations Using Massively Parallel Platforms," 44th AIAA Aerospace Sciences Meeting and Exhibit, AIAA Paper 20060421, Jan. 2006. doi: $10.2514 / 6.2006-421$

[35] Jameson, A., Schmidt, W., and Turkel, E., "Numerical Solution of the Euler Equations by Finite Volume Methods Using Runge Kutta Time Stepping Schemes," 14th Fluid and Plasma Dynamics Conference, AIAA Paper 1981-1259, 1981.

[36] Mader, C. A., Martins, J. R. R. A., Alonso, J. J., and van der Weide, E., "ADjoint: An Approach for the Rapid Development of Discrete Adjoint Solvers," AIAA Journal, Vol. 46, No. 4, 2008, pp. 863-873. doi: 10.2514/1.29123

[37] Saad, Y., and Schultz, M. H., "GMRES: A Generalized Minimal Residual Algorithm for Solving Nonsymmetric Linear Systems," SIAM Journal on Scientific and Statistical Computing, Vol. 7, No. 3, 1986, pp. 856-869. doi:10.1137/0907058

[38] Balay, S., Gropp, W. D., McInnes, L. C., and Smith, B. F., "Efficient Management of Parallelism in Object Oriented Numerical Software Libraries," Modern Software Tools in Scientific Computing, edited by Arge, E., Bruaset, A. M., and Langtangen, H. P., Birkhäuser, Boston, 1997, pp. 163-202. doi:10.1007/978-1-4612-1986-6_8

[39] Balay, S., Brown, J., Buschelman, K., Eijkhout, V., Gropp, W. D., Kaushik, D., Knepley, M. G., McInnes, L. C., Smith, B. F., and Zhang, 
H., "PETSc Users Manual," Rev. 3.4, Argonne National Lab., TR-ANL95/11, Lemont, IL, 2013.

[40] Balay, S., Brown, J., Buschelman, K., Gropp, W. D., Kaushik, D., Knepley, M. G., McInnes, L. C., Smith, B. F., and Zhang, H., "PETSc," Software Package, Argonne National Laboratory, Argonne, IL, 2013, http://www.mcs.anl.gov/petsc [retrieved 14 May 2014].

[41] Mader, C. A., and Martins, J. R. R. A., "Stability-Constrained Aerodynamic Shape Optimization of Flying Wings," Journal of Aircraft, Vol. 50, No. 5, 2013, pp. 1431-1449.

doi:10.2514/1.C031956

[42] Lyu, Z., and Martins, J. R. R. A., "Aerodynamic Shape Optimization of a Blended-Wing-Body Aircraft," 51 st AIAA Aerospace Sciences Meeting, AIAA Paper 2013-0283, Jan. 2013. doi: $10.2514 / 6.2013-283$

[43] Liem, R. P., Kenway, G. K. W., and Martins, J. R. R. A., "Combined Multi-Mission Aircraft Fuel Burn Minimization via Multi-Point Aerostructural Optimization," AIAA Journal (submitted for publication).

[44] Gill, P. E., Murray, W., and Saunders, M. A., "SNOPT: An SQP Algorithm for Large-Scale Constrained Optimization," SIAM Journal on Optimization, Vol. 12, No. 4, 2002, pp. 979-1006. doi: $10.1137 / \mathrm{S} 1052623499350013$

[45] Perez, R. E., Jansen, P. W., and Martins, J. R. R. A., "pyOpt: A PythonBased Object-Oriented Framework for Nonlinear Constrained
Optimization," Structures and Multidisciplinary Optimization, Vol. 45, No. 1, 2012, pp. 101-118.

doi:10.1007/s00158-011-0666-3

[46] Gunston, B., Jackson, P., Bushell, S., and Willis, D., IHS Jane's All the World's Aircraft 2014-2015: Development \& Production, Jane's Information Group, London, 2014, pp. 775-781.

[47] Roache, P. J., "Verification of Codes and Calculations," AIAA Journal, Vol. 36, No. 5, 1998, pp. 696-702. doi: $10.2514 / 2.457$

[48] Gill, P. E., Murray, W., and Saunders, M. A., "User's Guide for SNOPT Version 7: Software for Large-Scale Nonlinear Programming," Systems Optimization Laboratory, Stanford Univ., Stanford, CA, 2006.

[49] Torenbeek, E., Advanced Aircraft Design: Conceptual Design, Technology and Optimization of Subsonic Civil Airplanes, Aerospace Series, Wiley, Chichester, England, 2013, pp. 23-27. doi:10.1002/9781118568101

[50] Roberson, W., Root, R., and Adams, D., "Fuel Conservation Strategies: Cruise Flight,” AERO, Boeing AERO Magazine, QTR_4.07, 2007.

W. K. Anderson Associate Editor 
This article has been cited by:

1. Zhong-Hua Han, Yu Zhang, Chen-Xing Song, Ke-Shi Zhang. 2017. Weighted Gradient-Enhanced Kriging for HighDimensional Surrogate Modeling and Design Optimization. AIAA Journal 55:12, 4330-4346. [Abstract] [Full Text] [PDF] [PDF Plus]

2. James G. Coder, David Hue, Gaetan Kenway, Thomas H. Pulliam, Anthony J. Sclafani, Leonel Serrano, John C. Vassberg. Contributions to the Sixth Drag Prediction Workshop Using Structured, Overset Grid Methods. Journal of Aircraft, ahead of print1-14. [Abstract] [Full Text] [PDF] [PDF Plus]

3. Xingzhi Hu, Zhu Zhou, Xiaoqian Chen, Geoffrey T. Parks. Chance-Constrained Optimization Approach Based on Density Matching and Active Subspaces. ALAA Journal, ahead of print1-12. [Abstract] [Full Text] [PDF] [PDF Plus]

4. Sahuck Oh, Chung-Hsiang Jiang, Chiyu Jiang, Philip S. Marcus. 2017. Finding the optimal shape of the leading-andtrailing car of a high-speed train using design-by-morphing. Computational Mechanics 48. . [Crossref]

5. Shenren Xu, Sebastian Timme, Orest Mykhaskiv, Jens-Dominik Müller. 2017. Wing-body junction optimisation with CAD-based parametrisation including a moving intersection. Aerospace Science and Tecbnology 68, 543-551. [Crossref]

6. Justin Gray, Jeffrey Chin, Tristan Hearn, Eric Hendricks, Thomas Lavelle, Joaquim R. R. A. Martins. 2017. ChemicalEquilibrium Analysis with Adjoint Derivatives for Propulsion Cycle Analysis. Journal of Propulsion and Power 33:5, 1041-1052. [Abstract] [Full Text] [PDF] [PDF Plus]

7. Bijan Mohammadi. 2017. Controlling first four moments for robust optimization. Optimization and Engineering 18:3, 561-585. [Crossref]

8. S.N. Skinner, H. Zare-Behtash. 2017. State-of-the-art in aerodynamic shape optimisation methods. Applied Soft Computing . [Crossref]

9. Nathalie Bartoli, Thierry Lefebvre, Sylvain Dubreuil, Romain Olivanti, Nicolas Bons, Joaquim Martins, Mohamed-Amine Bouhlel, Joseph Morlier. An adaptive optimization strategy based on mixture of experts for wing aerodynamic design optimization . [Citation] [PDF] [PDF Plus]

10. Timothy R. Brooks, Gaetan K. Kenway, Joaquim Martins. Undeflected Common Research Model (uCRM): An Aerostructural Model for the Study of High Aspect Ratio Transport Aircraft Wings . [Citation] [PDF] [PDF Plus]

11. Orest Mykhaskiv, Pavanakumar Mohanamuraly, Jens-Dominik Mueller, Shenren Xu, Sebastian Timme. CAD-based shape optimisation of the NASA CRM wing-body intersection using differentiated CAD-kernel . [Citation] [PDF] [PDF Plus]

12. Nicolas Bons, Xiaolong He, Charles A. Mader, Joaquim Martins. Multimodality in Aerodynamic Wing Design Optimization . [Citation] [PDF] [PDF Plus]

13. Daniel J. Poole, Christian B. Allen, T. Rendall. Global Optimization of Multimodal Aerodynamic Optimization Benchmark Case . [Citation] [PDF] [PDF Plus]

14. Gaetan K. W. Kenway, Joaquim R. R. A. Martins. 2017. Buffet-Onset Constraint Formulation for Aerodynamic Shape Optimization. AIAA Journal 55:6, 1930-1947. [Abstract] [Full Text] [PDF] [PDF Plus]

15. Tristan Dhert, Turaj Ashuri, Joaquim R. R. A. Martins. 2017. Aerodynamic shape optimization of wind turbine blades using a Reynolds-averaged Navier-Stokes model and an adjoint method. Wind Energy 20:5, 909-926. [Crossref]

16. Nitin Garg, Gaetan K.W. Kenway, Joaquim R.R.A. Martins, Yin Lu Young. 2017. High-fidelity multipoint hydrostructural optimization of a 3-D hydrofoil. Journal of Fluids and Structures 71, 15-39. [Crossref]

17. Rhea P. Liem, Joaquim R.R.A. Martins, Gaetan K.W. Kenway. 2017. Expected drag minimization for aerodynamic design optimization based on aircraft operational data. Aerospace Science and Technology 63, 344-362. [Crossref]

18. Shenren Xu, Sebastian Timme. 2017. Robust and efficient adjoint solver for complex flow conditions. Computers \& Fluids 148, 26-38. [Crossref]

19. James G. Coder, Thomas H. Pulliam, David Hue, Gaetan K. Kenway, Anthony J. Sclafani. Contributions to the 6th AIAA CFD Drag Prediction Workshop Using Structured Grid Methods . [Citation] [PDF] [PDF Plus]

20. Daniel J. Poole, Christian B. Allen, T. Rendall. Objective Function and Constraints for Robust Transonic Aerofoil Optimization . [Citation] [PDF] [PDF Plus]

21. Gaetan K. Kenway, Asitav Mishra, Ney R. Secco, Karthikeyan Duraisamy, Joaquim Martins. An Efficient Parallel Overset Method for Aerodynamic Shape Optimization . [Citation] [PDF] [PDF Plus] 
22. Joshua Hodson, Douglas F. Hunsaker, Robert Spall. Wing Optimization using Dual Number Automatic Differentiation in MachUp . [Citation] [PDF] [PDF Plus]

23. D.J. Poole, C.B. Allen, T.C.S. Rendall. 2017. High-fidelity aerodynamic shape optimization using efficient orthogonal modal design variables with a constrained global optimizer. Computers \& Fluids 143, 1-15. [Crossref]

24. Christopher Lee, David Koo, David W. Zingg. 2017. Comparison of B-Spline Surface and Free-Form Deformation Geometry Control for Aerodynamic Optimization. ALAA Journal 55:1, 228-240. [Abstract] [Full Text] [PDF] [PDF Plus]

25. Weihua Su, Sean Shan-Min Swei, Guoming G. Zhu. 2016. Optimum Wing Shape of Highly Flexible Morphing Aircraft for Improved Flight Performance. Journal of Aircraft 53:5, 1305-1316. [Abstract] [Full Text] [PDF] [PDF Plus]

26. Christian B. Allen, Daniel J. Poole, T. Rendall. Efficient Modal Design Variables Applied to Aerodynamic Optimization of a Modern Transport Wing . [Citation] [PDF] [PDF Plus]

27. Andrea Viti, Thierry Druot, Antoine Dumont. Aero-structural approach coupled with direct operative cost optimization for new aircraft concept in preliminary design . [Citation] [PDF] [PDF Plus]

28. David A. Burdette, Gaetan K. Kenway, Joaquim Martins. Aerostructural design optimization of a continuous morphing trailing edge aircraft for improved mission performance . [Citation] [PDF] [PDF Plus]

29. Sylvain Arreckx, Andrew Lambe, Joaquim R. R. A. Martins, Dominique Orban. 2016. A matrix-free augmented lagrangian algorithm with application to large-scale structural design optimization. Optimization and Engineering 17:2, 359-384. [Crossref]

30. Weihua Su, Sean Shan-Min Swei, Guoming G. Zhu. Mission Adaptive Wing Shape Determination for Highly Flexible Aeroelastic Aircraft . [Citation] [PDF] [PDF Plus]

31. David A. Burdette, Gaetan K. Kenway, Joaquim Martins. Performance Evaluation of a Morphing Trailing Edge Using Multipoint Aerostructural Design Optimization . [Citation] [PDF] [PDF Plus]

32. Enrico Fabiano, Asitav Mishra, Dimitri J. Mavriplis, Karthik Mani. Time-dependent Aero-acoustic Adjoint-based Shape Optimization of Helicopter Rotors in Forward Flight . [Citation] [PDF] [PDF Plus]

33. Gaetan K. Kenway, Joaquim Martins. Aerodynamic Shape Optimization of the CRM Configuration Including BuffetOnset Conditions . [Citation] [PDF] [PDF Plus]

34. John Hwang, Joaquim Martins. Allocation-mission-design optimization of next-generation aircraft using a parallel computational framework . [Citation] [PDF] [PDF Plus]

35. Michael Meheut, Antoine Dumont, Gerald Carrier, Jacques E. Peter. Gradient-Based Optimization of CRM Wing-alone and Wing-body-tail Configurations by RANS Adjoint Technique . [Citation] [PDF] [PDF Plus]

36. Gaetan K. W. Kenway, Joaquim R. R. A. Martins. 2016. Multipoint Aerodynamic Shape Optimization Investigations of the Common Research Model Wing. AIAA Journal 54:1, 113-128. [Abstract] [Full Text] [PDF] [PDF Plus] [Supplemental Material]

37. Song Chen, Zhoujie Lyu, Gaetan K. W. Kenway, Joaquim R. R. A. Martins. 2016. Aerodynamic Shape Optimization of Common Research Model Wing-Body-Tail Configuration. Journal of Aircraft 53:1, 276-293. [Abstract] [Full Text] [PDF] [PDF Plus]

38. Zhoujie Lyu, Joaquim R. R. A. Martins. 2015. Aerodynamic Shape Optimization of an Adaptive Morphing Trailing-Edge Wing. Journal of Aircraft 52:6, 1951-1970. [Abstract] [Full Text] [PDF] [PDF Plus]

39. Gaetan K. Kenway, Joaquim Martins. High-Fidelity Aerostructural Otimization Considering Buffet Onset . [Citation] [PDF] [PDF Plus]

40. Daniel J. Poole, Christian B. Allen, T. Rendall. Free-Form Aerodynamic Wing Optimization Using MathematicallyDerived Design Variables . [Citation] [PDF] [PDF Plus]

41. Daniel J. Poole, Christian B. Allen, T. Rendall. Optimal Domain Element Shapes for Free-Form Aerodynamic Shape Control . [Citation] [PDF] [PDF Plus] 